\title{
CFT data in the Gross-Neveu model
}

\author{
Mikhail Goykhman ${ }^{*}$ and Ritam Sinha $\odot^{\dagger}$ \\ The Racah Institute of Physics, The Hebrew University of Jerusalem, Jerusalem 91904, Israel
}

(Received 24 November 2020; accepted 13 May 2021; published 7 June 2021)

\begin{abstract}
We calculate conformal field theory data for the Gross-Neveu model in $2<d<4$ dimensions at the nextto-leading order in the $1 / N$ expansion. In particular, we make use of the background field method to derive various conformal triangles involving the composite operator $s^{2}$, for the Hubbard-Stratonovich field $s$. We then apply these conformal triangles to obtain the corresponding one-pion exchange coefficients.
\end{abstract}

DOI: 10.1103/PhysRevD.103.125004

\section{INTRODUCTION}

Some of the most interesting phenomena in nature are associated with the regime of the strong interaction and therefore are not accessible to the standard perturbative treatment. Particularly, strongly-coupled physics occurs in the critical regime and is described by conformal field theories (CFTs) [1]. From the standpoint of fundamental physics, such a regime is sometimes reached at the end of the renormalization group flow of a quantum field theory, either in the deep infrared (IR) or in the asymptotically ultraviolet (UV) regime. Moreover, different systems can have their RG flows terminate at the same CFT, in a manifestation of the renowned phenomenon of critical universality.

The universality principle served as one of the inspirations to classify CFTs based on the algebra of primary operators and their observable properties, such as scaling dimensions and operator product expansion (OPE) coefficients, without necessarily specifying the Lagrangian of the underlying theory. In particular, the microscopic specifics of the underlying theory are ignored while the symmetries of the system and general consistency conditions (such as unitarity) constraining the available space of parameters come forward. The corresponding program is known as the conformal bootstrap, and it has been under active development over recent years [2-6].

This paper is motivated by the desire to expand our understanding of critical dynamics in $d=3$ dimensions. The well-known example of a three-dimensional critical system is furnished by the IR fixed point of the $O(n)$ vector model. This model can be viewed as a continuum limit

\footnotetext{
*michael.goykhman@mail.huji.ac.il

†ritam.sinha@mail.huji.ac.il
}

Published by the American Physical Society under the terms of the Creative Commons Attribution 4.0 International license. Further distribution of this work must maintain attribution to the author(s) and the published article's title, journal citation, and DOI. Funded by SCOAP ${ }^{3}$. description of the critical $n$-vector model on a lattice $[5,7,8]$. The latter in turn generalizes the three-dimensional Ising model, describing the second-order phase transition of a ferromagnet. The fermionic counterpart of the $O(n)$ vector model is given by the $U(n)$ models with quartic fermion couplings, which we choose as the main focus of this paper.

The fixed point of the $O(n)$ vector model exists in a perturbative Wilson-Fisher regime when the model is considered in $4-\epsilon$ dimensions for small values of $\epsilon$. The three-dimensional physics (after a proper resummation) is rather well approximated by setting $\epsilon=1$ [9]. Similarly, the $U(n)$ fermionic model with the four-fermion Gross-Neveu (GN) interaction is asymptotically free in two dimensions ${ }^{1}$ but possesses the Wilson-Fisher type of fixed point in the UV limit in $2+\epsilon$ dimensions $[10,11]^{2}$

While the critical vector models are nonperturbative in general $d$, a popular approach to study them is given by the $1 / N$ expansion, around an infinitely large number $N \rightarrow \infty$ of the degrees of freedom of the system (see [13] and references therein). The $1 / N$ expansion and conformal bootstrap techniques are well suited to study a strongly-coupled critical regime and have proven to be remarkably successful methods for extracting the CFT data such as the scaling dimensions and the OPE coefficients, see [11,14-44] for an incomplete list of related references. ${ }^{3}$

\footnotetext{
${ }^{1}$ In fact, it possesses a number of remarkable properties in two dimensions, such as the dynamical breaking of chiral symmetry and generation of the IR scale via the dimensional transmutation, making it a $2 \mathrm{~d}$ toy-model of quantum chromodynamics [10].

${ }^{2}$ See [12] for a recent discussion of the four-fermionic models in the strongly-coupled field theory.

${ }^{3}$ While the quartic coupling in the $O(n)$ vector model in $4<$ $d<6$ dimensions, and the GN coupling in $2<d<4$ dimensions are nonrenormalizable by power counting, these theories are renormalizable at each order in the $1 / N$ expansion $[13,45]$. See also [43] where consistency check for the existence of a fixed point in the $O(n)$ vector model in $2<d<6$ dimensions was carried out by examining the Callan-Symanzik equations.
} 
Recently the power of the background field method was emphasized in the context of large- $N$ vector models [44]. It was shown that formally fixing some of the degrees of freedom to their nondynamical background values provides a simple shortcut to the calculation of effective vertices at subleading orders in the $1 / N$ expansion. In particular, this procedure allows one to easily extract finite parts of the three-point correlation functions (OPE coefficients). This is contrasted with the typical focus of the research in large $N$ critical vector models on the calculation of the critical exponents. In the literature, the background field method has been used effectively for calculations in cases where the field acquires a vacuum expectation value (v.e.v.) as a result of spontaneous symmetry breaking [46]. However, in the case of [44], as well as in this paper, the considered fields do not necessarily acquire a v.e.v., and therefore the method should be viewed only as a calculation tool.

In this paper, we further establish the power of the background field method for the study of critical vector models. To this end, we will scrutinize the UV critical regime of the $U(n)$ fermionic model with the GN interaction, and compute the full $s^{2} s s$ and $s^{2} \bar{\psi} \psi$ effective vertices for the fermion $\psi$, the Hubbard-Stratonovich field $s$, and the composite operator $s^{2}$ in terms of the corresponding conformal triangles. ${ }^{4}$ This is the first time that the background field method is used to calculate correlation functions involving a composite operator. In a simple way, this method provides a very powerful technique to calculate the finite part of nonplanar diagrams contributing to these conformal triangles. We further use these conformal triangles to calculate the OPE coefficients appearing in the correlation functions involving the operator $s^{2}$, including the two-point function $\left\langle s^{2} s^{2}\right\rangle$, and the three-point functions $\left\langle s^{2} \bar{\psi} \psi\right\rangle$ and $\left\langle s^{2} s s\right\rangle$.

It is well known that the UV fixed point of the GN model in $2<d<4$ dimensions is described by the same CFT as the IR fixed point of the Gross-Neveu-Yukawa (GNY) model [11]. Moreover, the GNY model can be studied perturbatively in $d=4-\epsilon$ dimensions, and the corresponding CFT data should match the CFT data of the GN model [11]. We perform such a consistency check on all of our results obtained in this paper. ${ }^{5}$

The rest of this paper is organized as follows: in Sec. II we set up our conventions and review the known results in the literature that will be useful for the purposes of this

\footnotetext{
${ }^{4}$ See [22] for the earlier calculation of the $s^{2} s s$ conformal triangle, which focused on its singular part for the purpose of calculating the anomalous dimension $\gamma_{s^{2}}$. We extend their result by adding the finite part of this conformal triangle, essential for the calculation of the OPE coefficients.

${ }^{5}$ In a similar spirit, the $O(N)$ vector model in $4<d<6$ dimensions has been conjectured to have a UV completion in terms of the vector model coupled to a dynamical scalar field with cubic interaction. This conjecture has received numerous verifications up to the fourth order in perturbation theory [31-33,35,42,43].
}

paper. In Sec. III we use the background field method to calculate the new conformal triangles up to the next-toleading order in the $1 / N$ expansion. We derive the $s^{2} \bar{\psi} \psi$ conformal triangle in Sec. III A, and the $s^{2} s s$ conformal triangle in Sec. III B. In Sec. IV we carry out the calculation of the $\left\langle s^{2} s s\right\rangle$ three-point function at the next-to-leading order in the $1 / N$ expansion. In the process, we derive various OPE coefficients. In Sec. $\mathrm{V}$ we calculate the $\left\langle s^{2} \bar{\psi} \psi\right\rangle$ three-point function at the next-to-leading order in the $1 / N$ expansion. In Sec. VI we demonstrate how the $s^{2} s s$ conformal triangle can be calculated from the $\left\langle s^{2} s^{2}\right\rangle$ two-point function, which in particular serves as a nontrivial consistency check for our results. We conclude with the discussion in Sec. VII, where we also outline possible future directions.

\section{SETUP}

Consider the $U(n)$-invariant fermionic model in $2 \leq d \leq 4$ dimensions with the quartic GN interaction,

$$
S=\int d^{d} x\left(\bar{\psi} \gamma^{\mu} \partial_{\mu} \psi+\frac{g}{N}(\bar{\psi} \psi)^{2}\right)
$$

where $\psi$ is the $n$-component multiplet of Dirac fermions. According to the standard conventions $N=n \operatorname{tr} \mathbb{I}$, where $\mathbb{I}$ is the unit matrix in the $2^{[d / 2]}$-dimensional space of Dirac spinors. We will be working in the Euclidean signature, with Hermitian gamma-matrices, $\left(\gamma^{\mu}\right)^{\dagger}=\gamma^{\mu}$, such that $\left\{\gamma^{\mu}, \gamma^{\nu}\right\}=2 \delta^{\mu \nu} \mathbb{I}$. Below in this paper we will skip keeping explicit track of the $U(n)$ indices where it does not cause a confusion. ${ }^{6}$

Using the standard trick, known as the HubbardStratonovich transformation, we can rewrite the action (2.1) in terms of the original fermions $\psi$ as well as an auxiliary scalar field $s$,

$$
S=\int d^{d} x\left(\bar{\psi} \gamma^{\mu} \partial_{\mu} \psi-\frac{1}{4 g} s^{2}+\frac{1}{\sqrt{N}} s \bar{\psi} \psi\right)
$$

The Hubbard-Stratonovich field $s$ becomes dynamical due to the fermion loop diagrams. The model (2.2) is believed to reach a nontrivial UV fixed point in $2<d<4$ dimensions $[11],{ }^{7}$ and we will be studying the corresponding CFT.

We will use the following Feynman rules for the bare propagators of the Dirac fermion and the HubbardStratonovich field $s$, and the leading order interaction vertex,

\footnotetext{
${ }^{6}$ In particular, when writing down the Feynman rules, we will omit the Kronecker delta symbols for the $U(n)$ indices.

${ }^{7}$ Such a fixed point can be studied perturbatively in $2+\epsilon$ dimensions.
} 

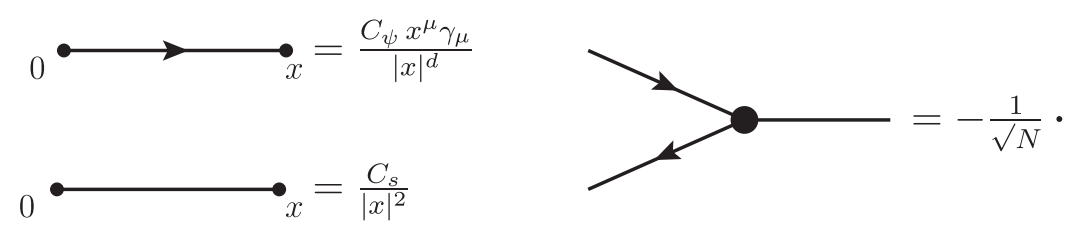

A fermionic loop generates the factor of $-n \operatorname{tr}(\mathbb{I})=-N$, where the minus sign appears as a consequence of the Wick contractions due to the anti-commuting nature of the fermion field. We will also use the following notation for the regularized Hubbard-Stratonovich propagator:

$$
0 \stackrel{2 \Delta_{s}+\delta}{\longrightarrow} \cdot \frac{C_{s} \mu^{-\delta}}{|x|^{2 \Delta_{s}+\delta}},
$$

where $\delta$ is the regularization parameter which is taken to zero at the end of calculation [16], $\mu$ is the renormalization mass scale, and $\Delta_{s}=1+\gamma_{s}$ is dimension of the Hubbard-Stratonovich field, which acquires the anomalous term $\gamma_{s}$. Our notations for the scalar and fermion lines with a general exponent and a unit amplitude will be as follows:

$$
0 \stackrel{2 a}{\longrightarrow}: \frac{x^{\mu} \gamma_{\mu}}{|x|^{2 a+1}} \quad 0 \stackrel{2 a}{\longrightarrow}{ }_{x}=\frac{1}{|x|^{2 a}}
$$

According to this notation, we will typically skip explicitly labeling exponents of the bare propagators of the fundamental fermion and the Hubbard-Stratonovich field.

The full fermion $\psi$ and Hubbard-Stratonovich $s$ propagators, including the anomalous dimensions $\gamma_{\psi, s}$ as well as corrections to the amplitudes $A_{\psi, s}$, will be denoted with solid blobs, and are given by

$$
\begin{aligned}
& \langle\psi(0) \bar{\psi}(x)\rangle=0 \longrightarrow{ }_{0}^{\bullet}=C_{\psi}\left(1+A_{\psi}\right) \mu^{-2 \gamma_{\psi}} \frac{x^{\mu} \gamma_{\mu}}{|x|^{d+2 \gamma_{\psi}}} \\
& \langle s(x) s(0)\rangle=0 \longrightarrow{ }_{x}^{\bullet}=C_{s}\left(1+A_{s}\right) \mu^{-2 \gamma_{s}} \frac{1}{|x|^{2+2 \gamma_{s}}}
\end{aligned}
$$

The full propagator of the composite field $s^{2}$ will be denoted as

$$
\left\langle s(x)^{2} s(0)^{2}\right\rangle=\square=C_{s^{2}}\left(1+A_{s^{2}}\right) \mu^{-2 \gamma_{s^{2}}} \frac{1}{|x|^{4+2 \gamma} s^{2}},
$$

where we defined

$$
C_{s^{2}}=2 C_{s}^{2}
$$

to be the leading order amplitude of the $\left\langle s^{2} s^{2}\right\rangle$ propagator. The leading order propagator amplitudes are given by [11]

$$
C_{\psi}=\frac{\Gamma\left(\frac{d}{2}\right)}{2 \pi^{\frac{d}{2}}}, \quad C_{s}=-\frac{2^{d} \sin \left(\frac{\pi d}{2}\right) \Gamma\left(\frac{d-1}{2}\right)}{\pi^{\frac{3}{2}} \Gamma\left(\frac{d}{2}-1\right)},
$$

The anomalous dimensions at the next-to-leading order are [11]

$$
\gamma_{\psi}=-\frac{1}{N} \frac{2^{d-1} \sin \left(\frac{\pi d}{2}\right) \Gamma\left(\frac{d-1}{2}\right)}{\pi^{\frac{3}{2}} d \Gamma\left(\frac{d}{2}-1\right)}+\mathcal{O}\left(1 / N^{2}\right), \quad \gamma_{s}=-4 \frac{d-1}{d-2} \gamma_{\psi}+\mathcal{O}\left(1 / N^{2}\right),
$$

and $1 / N$ corrections to the propagators amplitudes are [39] 


$$
A_{\psi}=-\frac{2}{d} \gamma_{\psi}+\mathcal{O}\left(1 / N^{2}\right), \quad A_{s}=-\left(H_{d-2}+\frac{2}{d}+\pi \cot \left(\frac{\pi d}{2}\right)\right) \gamma_{s}+\mathcal{O}\left(1 / N^{2}\right)
$$

where $H_{n}$ is $n$th harmonic number.

We will also need the $s \bar{\psi} \psi$ effective vertex, represented by the corresponding conformal triangle [1], and denoted with a solid blob

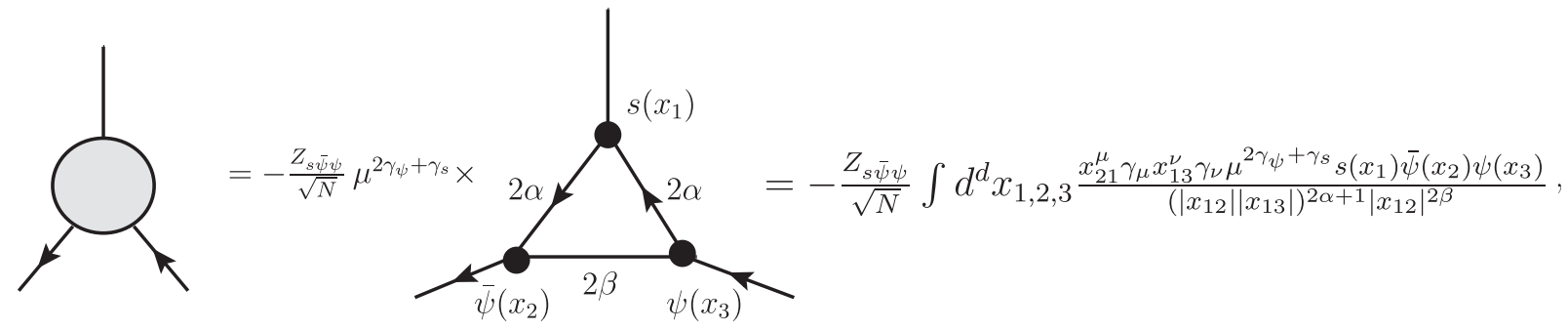

where the exponents

$$
\alpha=\frac{d-1-\gamma_{s}}{2}, \quad \beta=1-\gamma_{\psi}+\frac{\gamma_{s}}{2}
$$

are such that the integration vertices $x_{1,2,3}$ become unique when the propagators are attached to them. The $\bar{\psi} \psi s$ conformal triangle diagram is a sum of the leading order tree-level diagram, the subleading loop corrections, and the $\bar{\psi} \psi s$ vertex counterterm. In particular, the $\bar{\psi} \psi s$ vertex counterterm cancels the divergences from the subleading order loop diagram (see [44] for the detailed explanation).

To the next-to-leading order in $1 / N$ we can expand

$$
Z_{s \bar{\psi} \psi}=Z_{s \bar{\psi} \psi}^{(0)}\left(1+\delta Z_{s \bar{\psi} \psi}+\mathcal{O}\left(1 / N^{2}\right)\right),
$$

where $[39]^{8}$

$$
\begin{gathered}
Z_{s \bar{\psi} \psi}^{(0)}=-\frac{(d-2) \Gamma\left(\frac{d}{2}\right)^{2}}{4 \pi^{d}}\left(2 \gamma_{\psi}+\gamma_{s}\right), \\
\delta Z_{s \bar{\psi} \psi}=-\frac{2}{d-2} \gamma_{s} .
\end{gathered}
$$

For the analysis of loop diagrams we will be using the following propagator merging relations [18]:

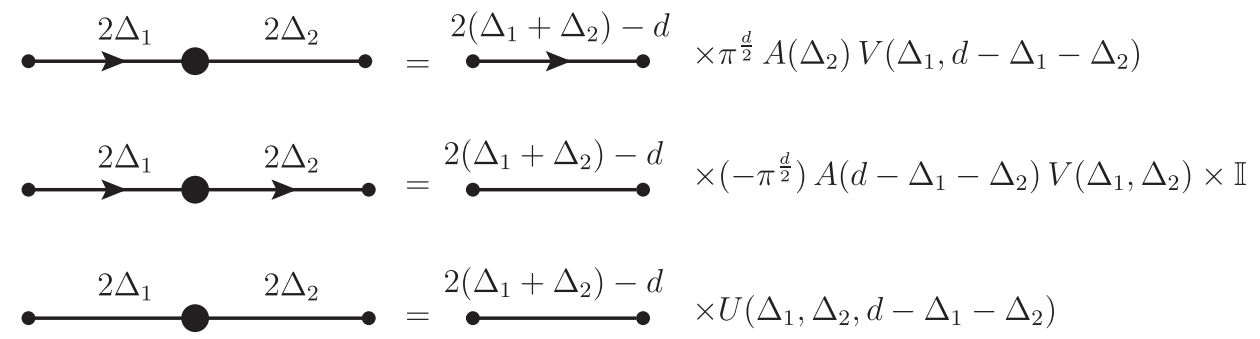

where we defined

$$
A(\Delta)=\frac{\Gamma\left(\frac{d}{2}-\Delta\right)}{\Gamma(\Delta)}, \quad V\left(\Delta_{1}, \Delta_{2}\right)=\frac{\Gamma\left(\frac{d+1}{2}-\Delta_{1}\right)}{\Gamma\left(\Delta_{1}+\frac{1}{2}\right)} \frac{\Gamma\left(\frac{d+1}{2}-\Delta_{2}\right)}{\Gamma\left(\Delta_{2}+\frac{1}{2}\right)}
$$

\footnotetext{
${ }^{8}$ See also [44] for a recent derivation.
} 


$$
U\left(\Delta_{1}, \Delta_{2}, \Delta_{2}\right)=\pi^{\frac{d}{2}} A\left(\Delta_{1}\right) A\left(\Delta_{2}\right) A\left(\Delta_{3}\right),
$$

and the uniqueness relations for $\Delta_{1}+\Delta_{2}+\Delta_{3}=d[18,47,48]$,

$$
\begin{gathered}
\int d^{d} x_{4} \frac{1}{\left|x_{14}\right|^{2 \Delta_{1}}\left|x_{24}\right|^{2 \Delta_{2}}\left|x_{34}\right|^{2 \Delta_{3}}}=\frac{U\left(\Delta_{1}, \Delta_{2}, \Delta_{3}\right)}{\left|x_{12}\right|^{d-2 \Delta_{3}}\left|x_{13}\right|^{d-2 \Delta_{2}}\left|x_{23}\right|^{d-2 \Delta_{1}}}, \\
\int d^{d} x_{4} \frac{x_{24}^{\mu} \gamma_{\mu} x_{41}^{\nu} \gamma_{\nu}}{\left|x_{14}\right|^{2 \Delta_{1}+1}\left|x_{24}\right|^{2 \Delta_{2}+1}\left|x_{34}\right|^{2 \Delta_{3}}}=\frac{\pi^{\frac{d}{2}} A\left(\Delta_{3}\right) V\left(\Delta_{1}, \Delta_{2}\right) x_{31}^{\mu} \gamma_{\mu} x_{23}^{\nu} \gamma_{\nu}}{\left|x_{12}\right|^{d-2 \Delta_{3}}\left|x_{13}\right|^{d-2 \Delta_{2}+1}\left|x_{23}\right|^{d-2 \Delta_{1}+1}} .
\end{gathered}
$$

Loops in position space are simply additive ${ }^{9}$

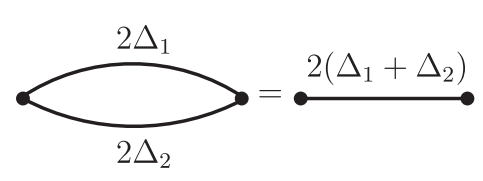

\section{CONFORMAL TRIANGLES}

The structure of three-point functions in CFTs is completely fixed by the conformal symmetry. It is usually convenient to decompose contributions to the three-point functions (and the corresponding OPE coefficients) into the terms originating from the corresponding conformal triangle [1], and the terms due to the amplitudes of the propagators attached to the conformal triangle [43]. In this section we will make use of the background field method to calculate the $s^{2} \bar{\psi} \psi$ and $s^{2} s s$ conformal triangles.

The results obtained in this section will be used below in Sec. IV to derive the $1 / N$ correction $A_{s^{2}}$ to the amplitude of the $\left\langle s^{2} s^{2}\right\rangle$ propagator, and the $1 / N$ corrections to the threepoint function $\left\langle s^{2} s s\right\rangle$. Finally, in Sec. V we will use these results to obtain the next-to-leading order corrections to the three-point function $\left\langle s^{2} \bar{\psi} \psi\right\rangle$.

\section{A. $s^{2} \overline{\boldsymbol{\psi}} \psi \boldsymbol{c o n f o r m a l}$ triangle}

The $s^{2} \bar{\psi} \psi$ conformal triangle can be represented diagrammatically as

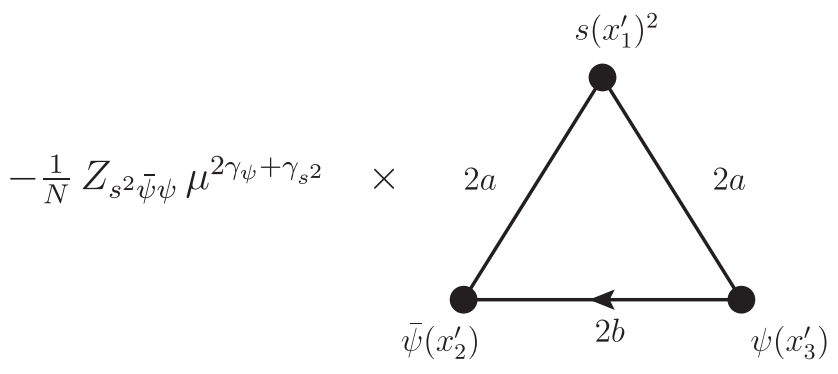

\footnotetext{
${ }^{9}$ Notice that the minus sign in the rhs of the last loop is independent of the minus sign in the factor of $-N$ appearing in the Feynman rule for the fermionic loop.
}

Here we have introduced

$$
a=\frac{d-\gamma_{s^{2}}}{2}-1, \quad b=\frac{3+\gamma_{s^{2}}}{2}-\gamma_{\psi}
$$

The structure of the conformal triangle is such that when the full propagators of the composite operator $s^{2}$ and the fermions $\psi$ are attached to it, the vertices $x_{1,2,3}^{\prime}$ of the triangle become unique and can be integrated over, resulting in the conformal three-point function

$$
\begin{aligned}
& \left\langle s^{2}\left(x_{1}\right) \bar{\psi}\left(x_{2}\right) \psi\left(x_{3}\right)\right\rangle \\
& \quad=C_{s^{2} \bar{\psi} \psi} \frac{\mu^{-2 \gamma_{\psi}-\gamma_{s^{2}}} x_{23}^{\mu} \gamma_{\mu}}{\left|x_{23}\right|^{d-2+2 \gamma_{\psi}-\gamma_{s^{2}}}\left(\left|x_{12}\right|\left|x_{13}\right|\right)^{2+\gamma_{s^{2}}}} .
\end{aligned}
$$

Here we have defined the amplitude coefficient

$$
C_{s^{2} \bar{\psi} \psi}=-\frac{1}{N} C_{s^{2}} C_{\psi}^{2}\left(1+A_{s^{2}}\right)\left(1+A_{\psi}\right)^{2} Z_{s^{2} \bar{\psi} \psi} \mathcal{U}
$$

where $C_{s^{2}}$ is given by (2.3), and $A_{s^{2}, \psi}$ are amplitude corrections to the $s^{2}, \psi$ propagators, and the factor of $\mathcal{U}$ originates from application of the uniqueness relations (2.13), (2.14)

$$
\begin{aligned}
\mathcal{U}= & -\pi^{d} U\left(2+\gamma_{s^{2}}, \frac{d-\gamma_{s^{2}}}{2}-1, \frac{d-\gamma_{s^{2}}}{2}-1\right) A\left(1+\frac{\gamma_{s^{2}}}{2}\right) \\
& \times A\left(\frac{d-\gamma_{s^{2}}}{2}-1\right) V\left(\frac{d-1-\gamma_{s^{2}}}{2}-\gamma_{\psi}, \frac{d-1}{2}+\gamma_{\psi}\right) \\
& \times V\left(\frac{d-1}{2}+\gamma_{\psi}, \frac{3+\gamma_{s^{2}}}{2}-\gamma_{\psi}\right) \\
= & U_{0}\left(1+\delta u+\mathcal{O}\left(\frac{1}{N^{2}}\right)\right),
\end{aligned}
$$


where

$$
\begin{gathered}
U_{0}=-\frac{2 \pi^{\frac{3 d}{2}}}{(d-4) \Gamma\left(\frac{d}{2}\right)^{3}}, \\
\delta u=\frac{2(d-4)^{2} \gamma_{\psi}-(3(d-8) d+40) \gamma_{s^{2}}}{2(d-4)(d-2)} .
\end{gathered}
$$

To separate out the leading and subleading contributions to the OPE coefficient, let us expand (3.3) in $1 / N$ as

$$
C_{s^{2} \bar{\psi} \psi}=C_{s^{2} \bar{\psi} \psi}^{(1 / N)}\left(1+\delta C_{s^{2} \bar{\psi} \psi}\right)
$$

At the leading order the three-point function $\left\langle s^{2} \bar{\psi} \psi\right\rangle$ is determined by the $\mathcal{O}(1 / N)$ diagram

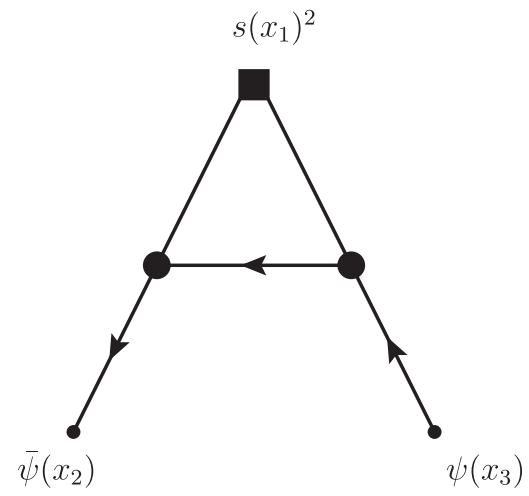

and was evaluated in [44]

$$
C_{s^{2} \bar{\psi} \mu}^{(1 / N)}=\frac{1}{N} \frac{2^{2 d-3} \pi^{-\frac{d}{2}-3}(\cos (\pi d)-1) \Gamma\left(\frac{d-1}{2}\right)^{2}}{\Gamma\left(\frac{d}{2}-1\right)} .
$$

Using this result we can determine the $s^{2} \bar{\psi} \psi$ conformal triangle amplitude

$$
Z_{s^{2} \bar{\psi} \psi}=Z_{s^{2} \bar{\psi} \psi}^{(0)}\left(1+\delta Z_{s^{2} \bar{\psi} \psi}\right)
$$

at the leading order in $1 / N$ as

$$
Z_{s^{2} \bar{\psi} \psi}^{(0)}=-N \frac{C_{s^{2} \bar{\psi} \psi}^{(1 / N)}}{C_{s^{2}} C_{\psi}^{2} U_{0}}=\frac{1}{2 \pi^{d}} \frac{4-d}{d-2} \Gamma\left(\frac{d}{2}\right)^{2} .
$$

We proceed to calculating the diagrams contributing to the conformal triangle amplitude correction $\delta Z_{s^{2} \bar{\psi} \psi}$ at the next-to-leading $\mathcal{O}(1 / N)$ order, which we represent as a sum of four terms. Diagrammatically, the conformal triangle up to the next-to-leading order in $1 / N$ is represented by the following equation:

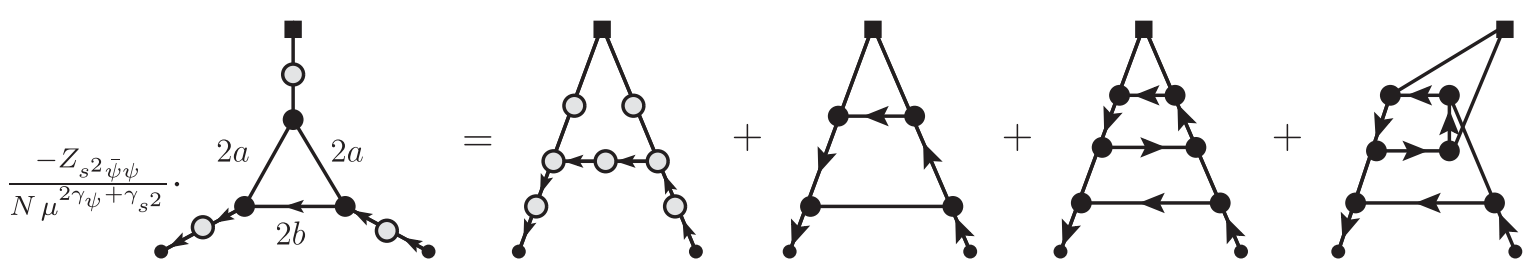

The gray blobs stand for the dressed $\psi$ and $s$ propagators and the dressed $s \bar{\psi} \psi$ vertex (represented by the corresponding $s \bar{\psi} \psi$ conformal triangle), according to the conventions introduced in Sec. II. We have also assigned the exponents of $2 a$ and $2 b$ to the internal lines of the $s^{2} \bar{\psi} \psi$ conformal triangle [see (3.1)].

A straightforward way to calculate the $\delta Z_{s^{2} \bar{\psi} \psi}$ is furnished by the background field method. ${ }^{10}$ To this end we split the Hubbard-Stratonovich field into the nondynamical background component $\bar{s}$ and the fluctuating component $s$, and isolate the $\mathcal{O}\left(\bar{s}^{2}\right)$ terms quadratic in the background $\bar{s}$. The resulting diagrammatic equation describes the fermionic propagator $\left.\langle\psi \bar{\psi}\rangle\right|_{\bar{s}}$ in the $\bar{s}$ background, at the second order in $\bar{s}$ :

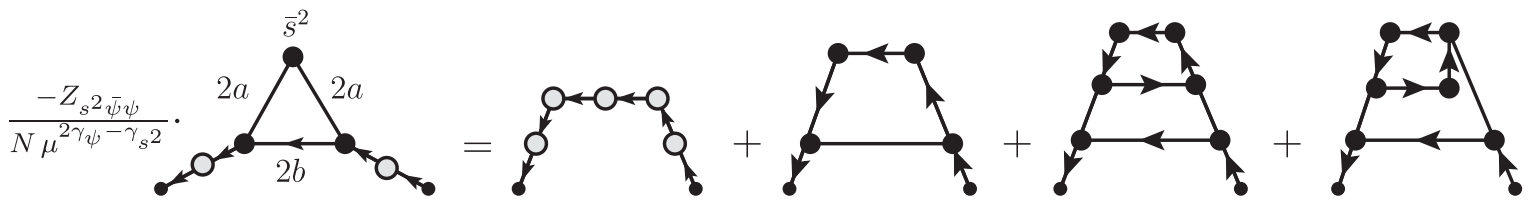

On the lhs of this equation we obtain

\footnotetext{
${ }^{10}$ See [44] where the background field method was first applied to obtain CFT data in vector models.
} 


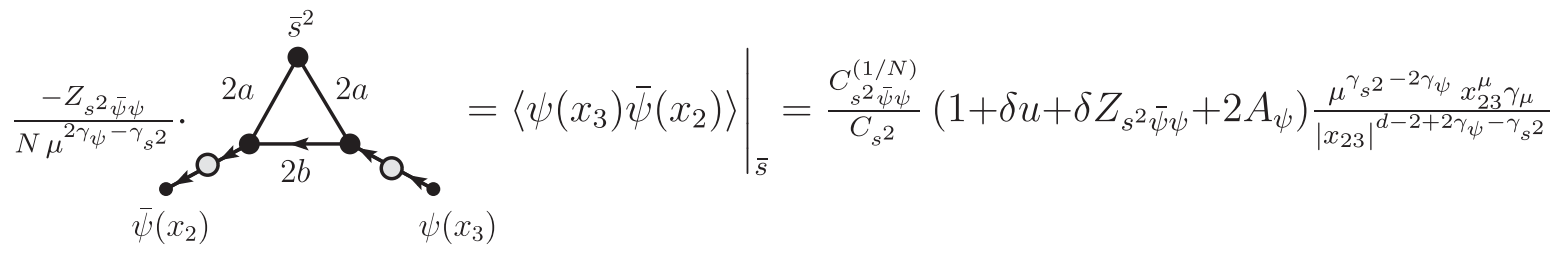

where we took into account (3.5), (3.6), (3.10), used the uniqueness relation (2.14), and linearized over the $1 / N$ corrections contributing to the overall amplitude.

We proceed to calculating the diagrams on the rhs of the diagrammatic equation for the $\langle\psi s \bar{\psi}\rangle_{\bar{s}}$. The first contribution is due to the leading order $\left\langle s^{2} \bar{\psi} \psi\right\rangle$ diagram in which all the internal $s \bar{\psi} \psi$ vertices and $\psi, s$ propagators have been dressed, and $s^{2}$ was set to the background value $\bar{s}^{2}$ :

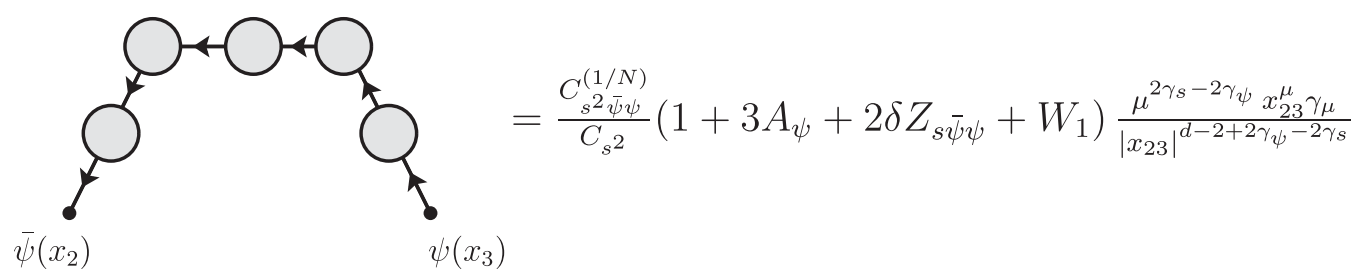

Notice that this diagram contains the entire leading order contribution to the $\left.\langle\psi \bar{\psi}\rangle\right|_{\bar{s}}$ two-point function. We linearized over the next-to-leading in $1 / N$ terms, obtaining the sum of $3 A_{\psi}$ due to amplitude corrections to three internal dressed fermion propagators and $2 \delta Z_{s \bar{\psi} \psi}$ due to two dressed $s \bar{\psi} \psi$ vertices. Finally, $W_{1}$ is obtained from the equation

$$
\begin{aligned}
1+W_{1}= & \frac{C_{s^{2}}}{C_{s^{2} \bar{\psi} \psi}^{(1 / N)}}\left(-\frac{Z_{s \bar{\psi} \psi \psi}^{(0)}}{\sqrt{N}}\right)^{2} C_{\psi}^{3}\left(-\pi^{2 d}\right) V\left(\frac{d-1}{2}+\gamma_{\psi}, \frac{d-1-\gamma_{s}}{2}\right)^{3} A\left(1-\gamma_{\psi}+\frac{\gamma_{s}}{2}\right)^{3} \\
& \times U\left(1+\gamma_{s}, \frac{d-\gamma_{s}}{2}-\gamma_{\psi}, \frac{d-\gamma_{s}}{2}-1+\gamma_{\psi}\right)^{2} A\left(\frac{d-\gamma_{s}}{2}-1+\gamma_{\psi}\right) V\left(\frac{d-1-\gamma_{s}}{2}, \frac{3}{2}-\gamma_{\psi}+\gamma_{s}\right),
\end{aligned}
$$

resulting from a repeated application of the uniqueness and the propagator merging relations, and expanded to the next-toleading order in $1 / N$. Further expanding the rhs in $\gamma_{\psi, s}=\mathcal{O}(1 / N)$ we obtain

$$
W_{1}=\frac{d-4}{d-2} \gamma_{\psi}+\frac{8-d}{d-2} \gamma_{s}
$$

Next, we consider the contribution represented by the diagram

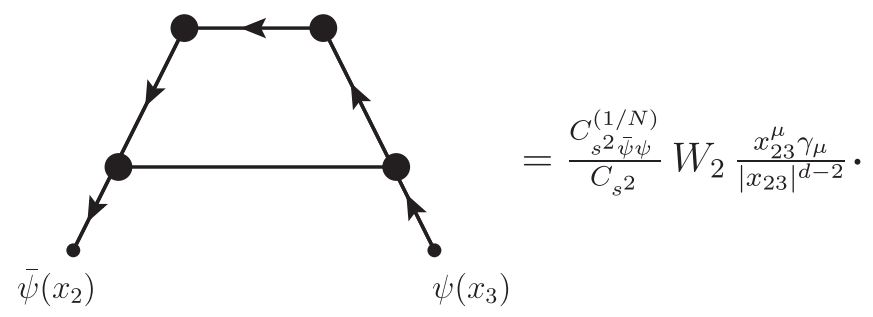

Repeated application of the propagator merging relations gives 


$$
\begin{aligned}
W_{2} & =\frac{C_{s^{2}}}{C_{s^{2}}^{(1 / N)}}\left(-\frac{1}{\sqrt{N} \psi}\right)^{4} C_{\psi}^{5} C_{s} \pi^{2 d} A(1)^{2} V\left(\frac{d-1}{2}, \frac{d-1}{2}\right)^{2} A\left(\frac{d}{2}-1\right)^{2} V\left(\frac{d-1}{2}, \frac{3}{2}\right)^{2} \\
& =\frac{1}{N} \frac{2^{d-2} \sin \left(\frac{\pi d}{2}\right) \Gamma\left(\frac{d-1}{2}\right)}{\pi^{3 / 2} \Gamma\left(\frac{d}{2}\right)} .
\end{aligned}
$$

The remaining contributions $W_{3,4}$ to the conformal triangle amplitude $\delta Z_{s^{2} \bar{\psi} \mu}$ originate from the next-to-leading corrections to the $\left\langle s^{2} s s\right\rangle$ subdiagram of the leading order $\left\langle s^{2} \bar{\psi} \psi\right\rangle$ diagram. To regularize these divergent diagrams we add a small correction $\delta / 2$ to the propagators of the internal $s$ lines, following the technique reviewed in Sec. II, ${ }^{11}$

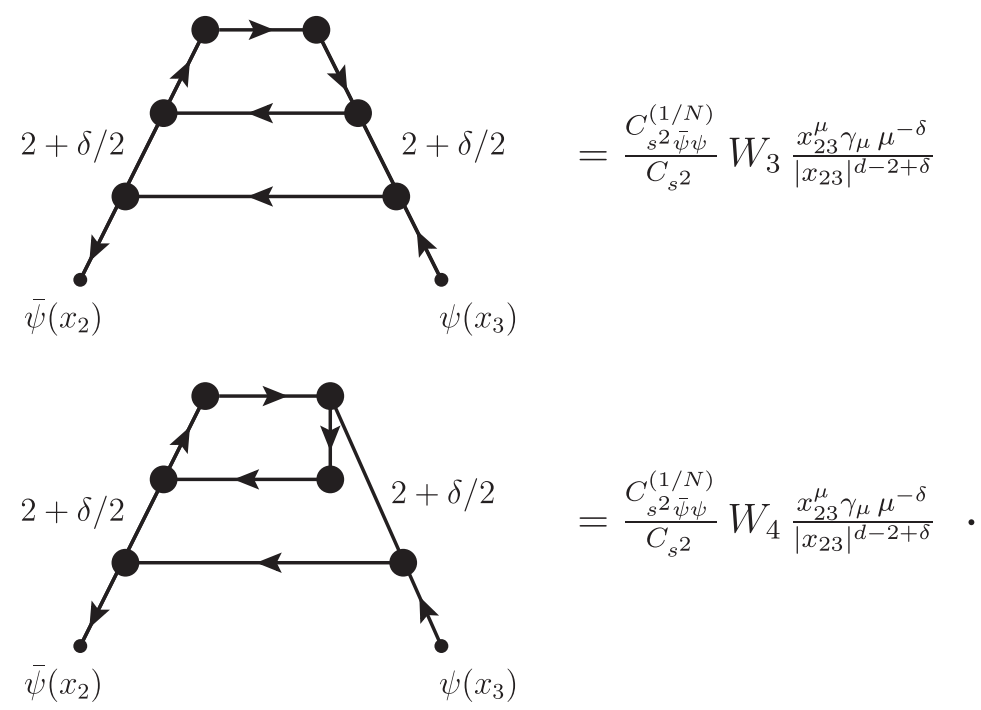

Here we have ${ }^{12}$

$$
\begin{aligned}
W_{3}= & -\frac{C_{s^{2}}}{C_{s^{2} \bar{\psi} \psi}^{(1 / N)}} 2 N\left(-\frac{1}{\sqrt{N}}\right)^{6} C_{\psi}^{7} C_{s}^{2}\left(-\pi^{2 d}\right) A(1) V\left(\frac{d-1}{2}, \frac{d-1}{2}\right) A\left(\frac{d}{2}-1\right) V\left(\frac{d-1}{2}, \frac{3}{2}\right) U\left(1+\frac{\delta}{4}, 1-\frac{\delta}{4}, d-2\right) \\
& \times U\left(1+\frac{\delta}{4}, \frac{2 d+\delta}{4}-1, \frac{d-\delta}{2}\right) A\left(1-\frac{\delta}{2}\right) V\left(\frac{d-1+\delta}{2}, \frac{d-1}{2}\right) A\left(\frac{d+\delta}{2}-1\right) V\left(\frac{d-1}{2}, \frac{3-\delta}{2}\right) \\
= & \frac{1}{N} \frac{2(d-2) \sin \left(\frac{\pi d}{2}\right) \Gamma(d-1)}{\pi \Gamma\left(\frac{d}{2}\right)^{2}}\left(\frac{2}{\delta}+1\right),
\end{aligned}
$$

and

$$
W_{4}=\frac{1}{d-2} W_{3} .
$$

Finally, we notice that the divergent terms of the last two diagrams add up to

$$
W_{3}+W_{4} \supset \frac{2 \gamma_{s}-\gamma_{s^{2}}}{\delta},
$$

which is cancelled by the counterterm diagram

\footnotetext{
${ }^{11}$ The choice of $\delta / 2$ instead of $\delta$ is a convention to make the total power of the diagram add up to $\delta$.

${ }^{12}$ The overall minus sign is due to the Feynman rule for the fermion loop, see Sec. II, while 2 is the factor of symmetry.
} 


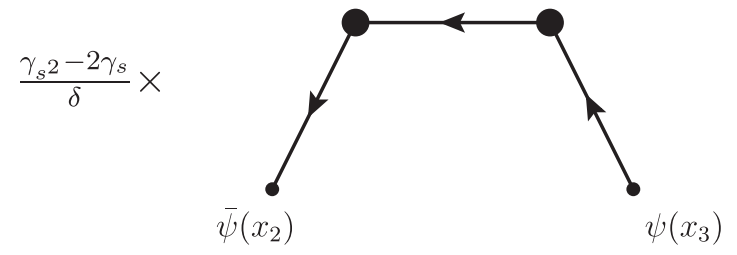

induced by the renormalization of the HubbardStratonovich field $s$ and the composite operator $s^{2}$

$$
s \rightarrow \sqrt{1+\frac{2 \gamma_{s}}{\delta}} s, \quad s^{2} \rightarrow \sqrt{1+\frac{2 \gamma_{s^{2}}}{\delta}} s^{2} .
$$

To summarize, we arrive at the following expression

$$
\delta Z_{s^{2} \bar{\psi} \psi}=\left.\sum_{i=1}^{4} W_{i}\right|_{\text {finite }}+2 \delta Z_{s \bar{\psi} \psi}+A_{\psi}-\delta u
$$

In what follows we will skip specifying explicitly that only the finite part of the sum of $W_{i}$ is retained, implying that the infinities have been cancelled out by the counterterm.

\section{B. $s^{2} s s$ conformal triangle}

In Sec. III A we demonstrated how the $\left\langle s^{2} \bar{\psi} \psi\right\rangle$ threepoint function can be expressed in terms of the corresponding $s^{2} \bar{\psi} \psi$ conformal triangle (3.3), and proceeded to calculate the latter, arriving at (3.17). The results of the previous section can in fact be used to calculate the $s^{2} s s$ conformal triangle at the next-to-leading order in $1 / N$, which we will use below to derive the $\left\langle s^{2} s s\right\rangle$ and $\left\langle s^{2} s^{2}\right\rangle$ correlation functions.

To this end, consider the dressed $s^{2} s s$ vertex expressed in terms of the corresponding conformal triangle
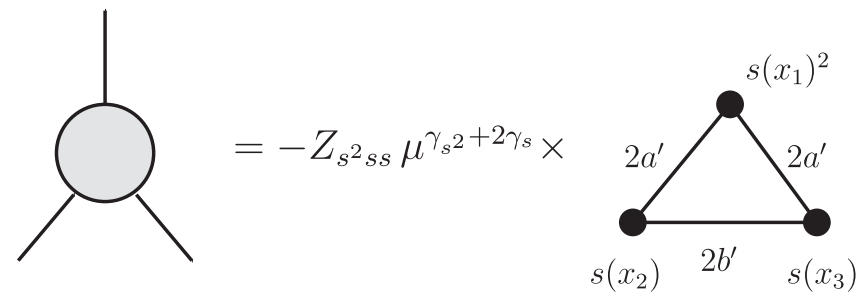

where we denoted

$$
a^{\prime}=\frac{d-\gamma_{s^{2}}}{2}-1, \quad b^{\prime}=\frac{d+\gamma_{s^{2}}}{2}-\gamma_{s}
$$

chosen so that when the $s^{2}$ and $s$ legs are attached to the triangle, its three vertices become unique and can be integrated over, resulting in

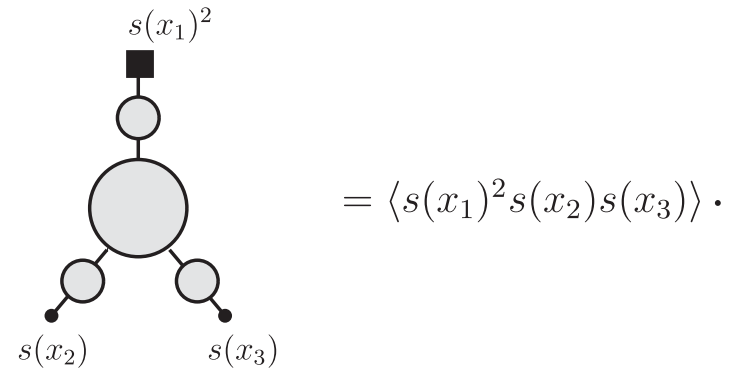

Conformal symmetry constrains the form of the $\left\langle s^{2} s s\right\rangle$ three-point to be

$\left\langle s\left(x_{1}\right)^{2} s\left(x_{2}\right) s\left(x_{3}\right)\right\rangle=\mu^{-\gamma_{s^{2}}-2 \gamma_{s}} \frac{C_{s^{2} s s}^{(0)}\left(1+\delta C_{s^{2} s s}\right)}{\left(\left|x_{12}\right|\left|x_{13}\right|\right)^{2+\gamma_{s^{2}}}\left|x_{23}\right|^{2 \gamma_{s}-\gamma_{s^{2}}}}$,

where

$$
C_{s^{2} s s}^{(0)}=2 C_{s}^{2}
$$

is the leading order OPE coefficient, $\delta C_{s^{2} s s}$ stands for the subleading corrections. The latter can be decomposed as

$$
\delta C_{s^{2} s s}=\delta V_{s^{2} s s}+A_{s^{2}}+2 A_{s},
$$

where the vertex amplitude correction $\delta V_{s^{2} s s}$ is defined by the diagram

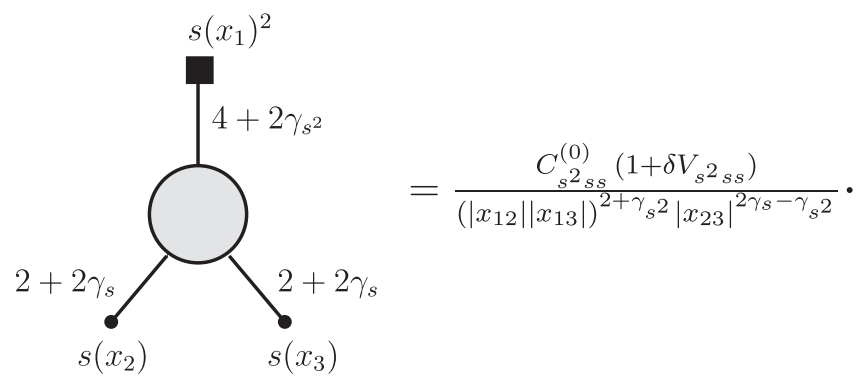

Using the uniqueness to integrate over three vertices of the conformal triangle we can obtain the relation between $C_{s^{2} s s}$ and $Z_{s^{2} s s}$,

$$
\begin{aligned}
C_{s^{2} s s}^{(0)}\left(1+\delta V_{s^{2} s s}\right) & \\
= & -Z_{s^{2} s s} C_{s}^{2} C_{s^{2}} U\left(\frac{d-\gamma_{s^{2}}}{2}-1, \frac{d-\gamma_{s^{2}}}{2}-1,2+\gamma_{s^{2}}\right) \\
& \times U\left(1+\frac{\gamma_{s^{2}}}{2}, 1+\gamma_{s}, d-2-\gamma_{s}-\frac{\gamma_{s^{2}}}{2}\right) \\
& \times U\left(1+\gamma_{s}, \frac{d+\gamma_{s^{2}}}{2}-\gamma_{s}, \frac{d-\gamma_{s^{2}}}{2}-1\right) .
\end{aligned}
$$

Expanding 


$$
Z_{s^{2} s s}=Z_{s^{2} s s}^{(0)}\left(1+\delta Z_{s^{2} s s}\right)
$$

we obtain

$$
\begin{gathered}
Z_{s^{2} s s}^{(0)}=\frac{(d-4) \pi^{3-\frac{3 d}{2}} \Gamma\left(\frac{d}{2}-1\right)^{2} \Gamma(d-1)}{2^{2 d+3} \Gamma\left(2-\frac{d}{2}\right) \Gamma\left(\frac{d-1}{2}\right)^{2} \sin ^{2}\left(\frac{\pi d}{2}\right)}\left(\gamma_{s^{2}}-2 \gamma_{s}\right), \\
\delta Z_{s^{2} s s}=\delta V_{s^{2} s s}-\delta z,
\end{gathered}
$$

where $^{13}$

$$
\begin{aligned}
\delta z= & \left(\frac{2}{d-2}+\pi \cot \left(\frac{\pi d}{2}\right)+\psi^{(0)}(d-2)+\gamma\right) \gamma_{s} \\
& +\frac{1}{2}\left(\frac{4}{d-4}-\frac{2}{d-2}+\pi \cot \left(\frac{\pi d}{2}\right)+\psi^{(0)}(d-2)+\gamma-2\right) \gamma_{s^{2}} .
\end{aligned}
$$

We can use the last two diagrams contributing to the $\langle\bar{\psi} \psi\rangle\rangle\left.\right|_{\bar{s}}$ correlation function, obtained in Sec. III A, to extract the next-to-leading order correction $\delta Z_{s^{2} s s}$ to the $s^{2} s s$ conformal triangle. The total of these diagrams is given by

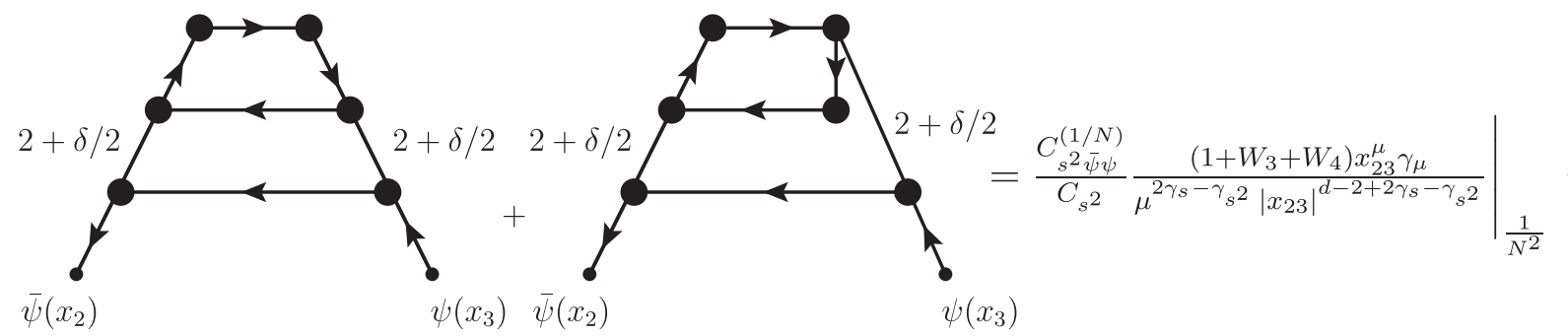

where in the rhs of this equation we only retain the subleading terms due to the amplitude $W_{3}+W_{4}$ and the anomalous dimensions $\gamma_{s, s^{2}}{ }^{14}$ On the other hand, we can rewrite these diagrams using the dressed $s^{2} s s$ vertex defined above

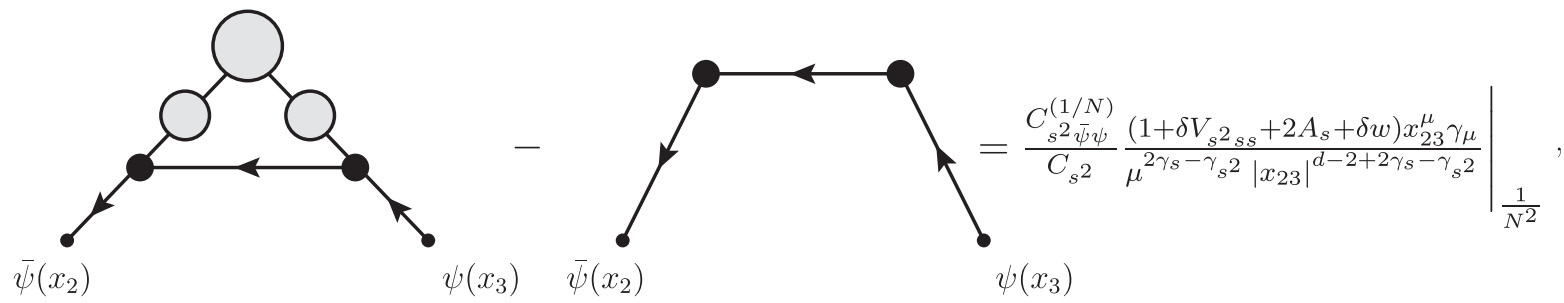

Here the extra term $\delta w$ originates as follows: Integrating over the unique vertices of the $s^{2} s s$ conformal triangle in the first diagram on the lhs of the last equation we obtain

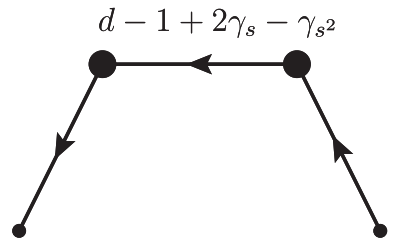

\footnotetext{
${ }^{13}$ Here $\gamma$ is the Euler constant, and we will denote the $n$th derivative of the digamma function $\psi^{(0)}(x)=\Gamma^{\prime}(x) / \Gamma(x)$ as $\psi^{(n)}(x)$.

${ }^{14}$ Linearizing over the $1 / N$ corrections, one can explicitly verify that the total of the four diagrams, considered in Sec. III A, contributing to the correlation function $\left.\langle\bar{\psi} \psi \psi\rangle\right|_{\bar{s}}$ at the next-to-leading order has the conformal form.
} 
Further taking the integrals over the last two vertices using the propagator merging relations, and expanding the result in $1 / N$ we arrive at

$$
\begin{aligned}
& \frac{C_{s^{2} \psi}^{(1 / N)}}{C_{s^{2}}}(1+\delta w) \\
& =\frac{C_{\psi}^{3}}{N}\left(-\pi^{d}\right) A\left(1-\gamma_{s}+\frac{\gamma_{s^{2}}}{2}\right) V\left(\frac{d-1}{2}, \frac{d-1-\gamma_{s^{2}}}{2}+\gamma_{s}\right) \\
& \quad \times A\left(\frac{d-\gamma_{s^{2}}}{2}-1+\gamma_{s}\right) V\left(\frac{d-1}{2}, \frac{3+\gamma_{s^{2}}}{2}-\gamma_{s}\right)
\end{aligned}
$$

where we defined

$$
\delta w=\frac{1}{2} \frac{d-4}{d-2}\left(2 \gamma_{s}-\gamma_{s^{2}}\right)
$$

Finally, we obtain

$$
\delta V_{s^{2} s s}=W_{3}+W_{4}-\delta w-2 A_{s}
$$

and therefore due to $(3.25)$

$$
\delta Z_{s^{2} s s}=W_{3}+W_{4}-\delta w-2 A_{s}-\delta z
$$

where $\delta z$ was calculated in (3.26).

$$
\text { IV. }\left\langle s^{2} s s\right\rangle
$$

In this section we are going to calculate the $\left\langle s^{2} s s\right\rangle$ and $\left\langle s^{2} s^{2}\right\rangle$ correlation functions at the next-to-leading order in the $1 / N$ expansion. In particular, we will obtain expression for the $1 / N$ correction $A_{s^{2}}$ to the propagator amplitude of the composite operator $s^{2}$. Besides deriving these new results, we will also reproduce the known expression for the anomalous dimension of the composite operator $s^{2}$.

Up to the next-to-leading order in $1 / N$, the effective $\left\langle s^{2} s s\right\rangle$ three-point function is determined by the following diagrams:

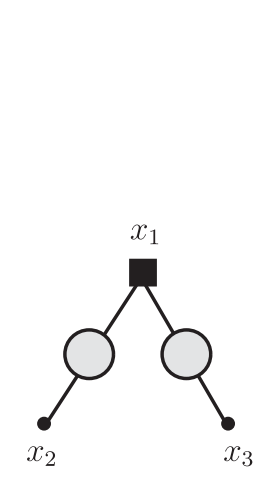

(a)

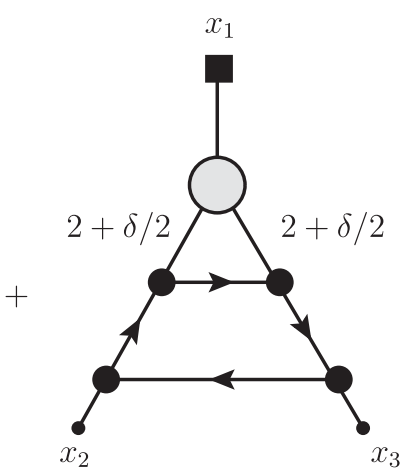

(b)

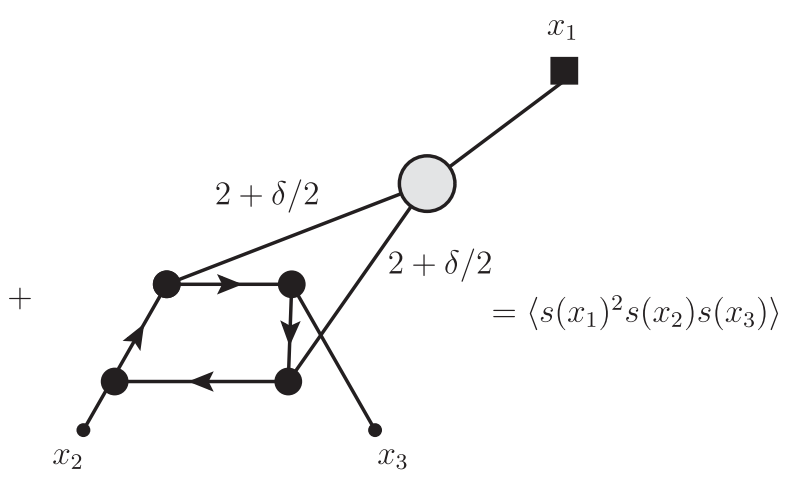

(c)
The diagram (a) of (4.1) represents contributions due to the leading-order $\left\langle s^{2} s s\right\rangle$ diagram where the $\langle s s\rangle$ propagators have been dressed. Recall that we denote the dressed propagators with a solid blob, according to the conventions introduced in Sec. II. In particular, this diagram includes the entire leading-order expression for the $\left\langle s^{2} s s\right\rangle$ three-point function.

The diagrams (b) and (c) of (4.1) are purely subleading in $1 / N$. Notice that in these diagrams we incorporated the $s^{2} s s$ conformal triangle [22] (denoted with a solid blob, following the conventions of Sec. III B, and regularized the inner $s$ propagators by a small additional exponent $\delta$. The latter will add important contributions to the finite part of the $\left\langle s^{2} s s\right\rangle$ three-point function, when we expand the $s^{2} s s$ conformal triangle subdiagram to the leading order in $1 / N$.

Renormalizing the Hubbard-Stratonovich field $s$ and the composite operator $s^{2}$ due to (3.16) induces the counterterm diagram

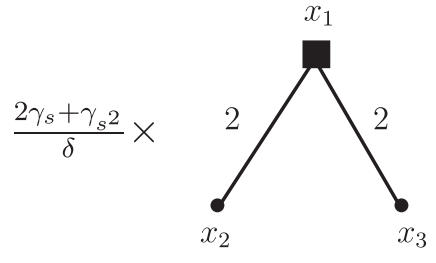

which will cancel the divergences.

We proceed with the calculation by integrating each term on both sides of (4.1) with respect to $x_{1}$. On the rhs we obtain

$$
\begin{aligned}
\int d^{d} x_{1}\left\langle s\left(x_{1}\right)^{2} s\left(x_{2}\right) s\left(x_{3}\right)\right\rangle \\
=2 C_{s}^{2} U(1,1, d-2)\left(1+h_{0}+\delta C_{s^{2} s s}\right) \\
\quad \times \frac{\mu^{-2 \gamma_{s}-\gamma_{s}^{2}}}{\left|x_{23}\right|^{4-d+2 \gamma_{s}+\gamma_{s}^{2}}},
\end{aligned}
$$


where expanding in $1 / N$ we get,

$$
\begin{aligned}
U\left(1+\frac{\gamma_{s^{2}}}{2}, 1+\frac{\gamma_{s^{2}}}{2}, d-2-\gamma_{s^{2}}\right) & =U(1,1, d-2)\left(1+h_{0}\right) \\
h_{0} & =\gamma_{s^{2}} r
\end{aligned}
$$

where $r$ is defined as

$$
r=H_{d-3}+\pi \cot \left(\frac{\pi d}{2}\right)
$$

while on the lhs of (4.1) we obtain the sum of three terms due to the corresponding contributing diagrams (a), (b), and (c), which we represent as

$$
\begin{aligned}
& \int d^{d} x_{1}\left\langle s\left(x_{1}\right)^{2} s\left(x_{2}\right) s\left(x_{3}\right)\right\rangle \\
& =\frac{2 C_{s}^{2} U(1,1, d-2)}{\left|x_{23}\right|^{4-d}}\left(\left[1+h_{1}+2 A_{s}-4 \gamma_{s} \log \left(\mu\left|x_{23}\right|\right)\right]\right. \\
& \left.\quad+\left[h_{2}-\omega_{2} \log \left(\mu\left|x_{23}\right|\right)\right]+\left[h_{3}-\omega_{3} \log \left(\mu\left|x_{23}\right|\right)\right]\right) .
\end{aligned}
$$

Here we grouped the terms in square brackets according to their origin from each of the three corresponding diagrams.

Notice that each diagram contributes a finite amplitude, which we denote as $h_{i}, i=2,3$, for the diagrams (b), (c), and $1+h_{1}+2 A_{s}$ for the diagram (a). We decomposed the $1 / N$ corrections to the latter into the contribution $2 A_{s}$ originating from the $1 / N$ correction to the amplitude of the $\langle s s\rangle$ propagators, as well as $h_{1}$ due to the $1 / N$ expansion

$$
\begin{aligned}
U\left(1+\gamma_{s}, 1+\gamma_{s}, d-2-2 \gamma_{s}\right) & =U(1,1, d-2)\left(1+h_{1}\right), \\
h_{1} & =2 \gamma_{s} r
\end{aligned}
$$

of the integral over $x_{1}$.

Besides the finite amplitudes, each term on the lhs of (4.1) contributes logarithmic terms due to the anomalous dimensions. Specifically, the diagram (a) contributes the coefficient of $-4 \gamma_{s}$, while we denoted contributions of the diagrams (b) and (c) respectively as $-\omega_{2}$ and $-\omega_{3}$. We will derive these expressions below in this section.

It is straightforward to calculate the integral over $x_{1}$ of the diagram (b) of (4.1). By repeatedly applying the uniqueness and propagator merging relations we obtain ${ }^{15}$

$$
\begin{aligned}
\int d^{d} x_{1} \frac{\left\langle s\left(x_{1}\right)^{2} s\left(x_{2}\right) s\left(x_{3}\right)\right\rangle}{2 C_{s}^{2} U(1,1, d-2)} \supset & \frac{1}{N}(-2) C_{s}^{2} C_{\psi}^{4}\left(1+\frac{r \delta}{2}\right) \pi^{d} A\left(\frac{d-\delta}{2}-1\right) V\left(\frac{3+\delta}{2}, \frac{d-1}{2}\right) A\left(1+\frac{\delta}{2}\right) V\left(\frac{d-1}{2}, \frac{d-1-\delta}{2}\right) \\
& \times U\left(\frac{d+\delta}{2}, \frac{d-\delta}{2}-1,1\right) U\left(1,1+\frac{\delta}{2}, d-2-\frac{\delta}{2}\right) \frac{\mu^{-\delta}}{\left|x_{23}\right|^{4-d+\delta}} .
\end{aligned}
$$

The factor of $1+r \delta / 2$, which we have already anticipated above, originates from the expansion to the linear order in $\delta$ of the integral over the position $x_{1}$ of the $s^{2}$ field, as well as the integral over the three vertices of the $s^{2} s s$ conformal triangle, while keeping only contributions at the leading order in $1 / N{ }^{16}$ Taking the limit $\delta \rightarrow 0$ we obtain

\footnotetext{
${ }^{15}$ The factor of -1 is due to the Feynman rule for the fermionic loop, and 2 is the symmetry factor. The sign $\supset$ indicates that the rhs of the equation gives one of the several terms contributing to the lhs, we use it to denote one of the contributing diagrams to the considered correlation function.

${ }^{16}$ The shortcut to reproduce $r$ is given by

$$
\begin{gathered}
A\left(1+\frac{\delta}{4}\right) A\left(\frac{2 d-\delta}{4}-1\right) U\left(1+\frac{\delta}{4}, 1+\frac{\delta}{4}, d-2-\frac{\delta}{2}\right) \\
=A(1) A\left(\frac{d}{2}-1\right) U(1,1, d-2)\left(1+\frac{r \delta}{2}\right),
\end{gathered}
$$

where we retained only the $\delta$-dependent factors of the functions originating from taking the conformal integrals.
}

$$
\begin{aligned}
\omega_{2}= & \frac{1}{N} \frac{2^{d}(1-\cos (\pi d)) \Gamma\left(2-\frac{d}{2}\right) \Gamma\left(\frac{d-1}{2}\right)}{\pi^{5 / 2}}, \\
h_{2}= & \frac{1}{N} 2^{d} \pi^{-\frac{5}{2}} \sin ^{2}\left(\frac{\pi d}{2}\right) \Gamma\left(2-\frac{d}{2}\right) \\
& \times \Gamma\left(\frac{d-1}{2}\right)(2 r-1) .
\end{aligned}
$$

Finally, we proceed to the calculation of the nonplanar diagram, given by the diagram (c) of (4.1). Integrating over $x_{1}$, the vertices of the conformal triangle, and two of the opposite $s \bar{\psi} \psi$ vertices we arrive at

$$
\begin{aligned}
\int d^{d} x_{1} & \frac{\left\langle s\left(x_{1}\right)^{2} s\left(x_{2}\right) s\left(x_{3}\right)\right\rangle}{2 C_{s}^{2} U(1,1, d-2)} \\
\supset & \frac{1}{N}(-1) C_{s}^{2} C_{\psi}^{4}\left(1+\frac{r \delta}{2}\right)\left(\pi^{\frac{d}{2}} A(1) V\left(\frac{d-1}{2}, \frac{d-1}{2}\right)\right)^{2} \\
& \times \frac{v \mu^{-\delta}}{|x|^{4-d+\delta}},
\end{aligned}
$$

where we defined $v$ as an amplitude of the graph 


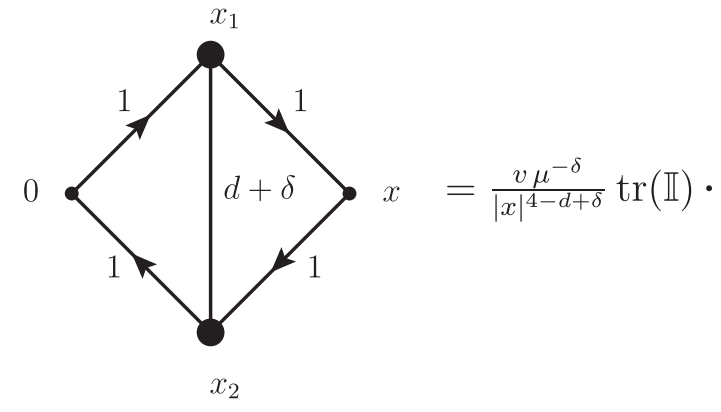

Using the standard expression for the trace of product of four gamma matrices, and manipulating some scalar products, we obtain

$$
\begin{aligned}
\frac{v}{|x|^{4-d+\delta}} & =\int d^{d} x_{1,2} \frac{1}{\left(\left|x_{1}\right|\left|x_{2}-x\right|\right)^{2}\left|x_{12}\right|^{d+\delta}} \\
& -\frac{|x|^{2}}{2} \int d^{d} x_{1,2} \frac{1}{\left(\left|x_{1}\right|\left|x_{1}-x\right|\left|x_{2}-x\right|\left|x_{2}\right|\right)^{2}\left|x_{12}\right|^{d-2+\delta}} .
\end{aligned}
$$

The first term in (4.12) can be evaluated using the propagator merging relations, while the second one can be reduced via inversion transformation around the lefthand external point to the $\operatorname{ChT}(1,1)$ integral, given by (16) in [15]. ${ }^{17}$ As a result we obtain

$$
\begin{aligned}
\frac{v \mu^{-\delta}}{|x|^{4-d+\delta}=} & {\left[\frac{U\left(\frac{d+\delta}{2}, \frac{d-\delta}{2}-1,1\right) U\left(1,1+\frac{\delta}{2}, d-2-\frac{\delta}{2}\right)}{\left(\mu\left|x_{23}\right|\right)^{\delta}}\right.} \\
& \left.-\frac{1}{2} \operatorname{ChT}(1,1)\right] \frac{1}{|x|^{4-d}},
\end{aligned}
$$

where

$\operatorname{ChT}(1,1)=\pi^{d} \cos \left(\frac{\pi d}{2}\right) \Gamma(3-d)\left(\pi^{2}-6 \psi^{(1)}\left(\frac{d}{2}-1\right)\right)$.

Interestingly, only the first term in (4.13) is divergent in the $\delta \rightarrow 0$ limit. Combining everything together we obtain

$$
\begin{gathered}
\omega_{3}=\frac{1}{d-2} \omega_{2} \\
h_{3}=\frac{1}{N} \frac{2^{d-4} \Gamma\left(\frac{d-1}{2}\right) \sin \left(\frac{\pi d}{2}\right)}{\pi^{3 / 2}(d-2) \Gamma\left(\frac{d}{2}\right)}\left(\pi^{2}(d-2)^{2}-16 \gamma(d-2)\right. \\
-16 \pi(d-2) \cot \left(\frac{\pi d}{2}\right) \\
-6(d-2)^{2} \psi^{(1)}\left(\frac{d}{2}-1\right) \\
\left.-16(d-2) \psi^{(0)}(d-2)+16\right) .
\end{gathered}
$$

Comparing the logarithmic terms on both sides of (4.1) we conclude

$$
\gamma_{s^{2}}=2 \gamma_{s}+\omega_{2}+\omega_{3}
$$

Using (2.5), (4.9), (4.15), (4.17) we therefore arrive at

$$
\gamma_{s^{2}}=(2-d) \gamma_{s}
$$

in agreement with the known result [18].

On the other hand, comparing the finite terms on both sides of (4.1) we get

$$
\delta C_{s^{2} s s}=h_{1}+2 A_{s}+h_{2}+h_{3}-h_{0} .
$$

Consequently due to (3.21) we derive

$$
A_{s^{2}}=h_{1}+h_{2}+h_{3}-h_{0}-\delta V_{s^{2} s s} .
$$

Using (3.30) we can rewrite it as

$$
A_{s^{2}}=2 A_{s}+h,
$$

where we introduced

$$
h=h_{1}+h_{2}+h_{3}-h_{0}-W_{3}-W_{4}+\delta w,
$$

which we can simplify as

$$
h=\frac{1}{N} \frac{2^{d-4} \sin \left(\frac{\pi d}{2}\right) \Gamma\left(\frac{d-1}{2}\right)\left(8(d-4)+\pi^{2}(d-2)-6(d-2) \psi^{(1)}\left(\frac{d}{2}-1\right)\right)}{\pi^{3 / 2} \Gamma\left(\frac{d}{2}\right)} .
$$

Above we have derived the next-to-leading order correction (4.19) to the amplitude of the three-point function $\left\langle s^{2} s s\right\rangle$ given by (3.19). It is useful to obtain the counterpart of this expression for the three-point function of the normalized fields,

$$
s \rightarrow \sqrt{C_{s}\left(1+A_{s}\right)} s, \quad s^{2} \rightarrow \sqrt{C_{s^{2}}\left(1+A_{s^{2}}\right)} s^{2} .
$$

\footnotetext{
${ }^{17}$ See also Appendix B in [43] for a review of the derivation of this integral.
} 
Using (3.21) we obtain

$$
\delta \hat{C}_{s^{2} s s}=\delta V_{s^{2} s s}+\frac{A_{s^{2}}}{2}+A_{s} .
$$

Plugging in expression (3.30) for $\delta V_{s^{2} s s}$ and (4.21) for $A_{s^{2}}$ we arrive at

$$
\delta \hat{C}_{s^{2} s s}=W_{3}+W_{4}-\delta w+\frac{h}{2} .
$$

We can carry out consistency checks of our result (4.26) by considering its limiting values in $d=2,4$ dimensions,

$$
\left.\delta \hat{C}_{s^{2} s s}\right|_{d=2}=-\frac{1}{2 N},\left.\quad \delta \hat{C}_{s^{2} s s}\right|_{d=4}=0 .
$$

Notice that in $d=2$ the theory is UV free, and therefore we have $s \simeq \bar{\psi}^{i} \psi^{i}$. By performing explicit contractions of the constituent fermions we obtain

$$
\begin{gathered}
\langle s(x) s(0)\rangle=2 N C_{\psi}^{2} \frac{1}{|x|^{2}}, \\
\left\langle s^{2}(x) s^{2}(0)\right\rangle=2 N^{2} C_{\psi}^{4}\left(1-\frac{1}{N}\right) \frac{1}{|x|^{4}}, \\
\left\langle s^{2}\left(x_{1}\right) s\left(x_{2}\right) s\left(x_{3}\right)\right\rangle=2 N^{2} C_{\psi}^{4}\left(1-\frac{1}{N}\right) \frac{1}{\left(\left|x_{12}\right|\left|x_{13}\right|\right)^{2}} .
\end{gathered}
$$

Therefore the next-to-leading in $1 / N$ correction to the normalized three-point function $\left\langle s^{2} s s\right\rangle$ is given by

$$
\delta \hat{C}_{s^{2} s s}=-\frac{1}{2 N},
$$

in agreement with (4.27).

At the same time, in $d=4-\epsilon$ dimensions the UV fixed point of the GN model is equivalent to the IR fixed point of the Gross-Neveu-Yukawa (GNY) model [11]. Such an equivalence implies that the CFT data of both critical theories must agree. In fact, for the GNY model, to the leading order in the $\epsilon$-expansion we obtain $\delta \hat{C}_{s^{2} s s}=0$, in agreement with (4.27).

\section{V. $\left\langle s^{2} \bar{\psi} \psi\right\rangle$}

In this section we are going to calculate the next-toleading order correction $\delta C_{s^{2} \bar{\psi} \psi}$ to the OPE coefficient $C_{s^{2} \bar{\psi} \psi}$. Due to (3.3) the total correction is given by the sum of the correction $\delta Z_{s^{2} \bar{\psi} \psi}$ to the amplitude of the $s^{2} \bar{\psi} \psi$ conformal triangle (3.9), as well as the amplitude corrections to the propagators of the fermion $\psi$ and the composite operator $s^{2}$ attached to the conformal triangle,

$$
C_{s^{2} \bar{\psi} \psi \psi}=C_{s^{2} \bar{\psi} \mu}^{(1 / N)}\left(1+\delta Z_{s^{2} \bar{\psi} \psi \psi}+2 A_{\psi}+A_{s^{2}}+\delta u+\mathcal{O}\left(\frac{1}{N^{2}}\right)\right),
$$

where $\delta u$ is given by (3.6), and originates from integrating over the vertices of the conformal triangle. When calculating the OPE coefficients it is conventional to rescale the external fields so that their propagators are normalized to unity. Thus, rescaling $s^{2}$ according to (4.24), and $\psi$ according to

$$
\psi \rightarrow \sqrt{C_{\psi}\left(1+A_{\psi}\right)} \psi
$$

we obtain the OPE coefficient for the normalized fields

$$
\begin{aligned}
\hat{C}_{s^{2} \bar{\psi} \psi \psi} & =\hat{C}_{s^{2} \bar{\psi} \psi}^{(1 / N)}\left(1+\delta \hat{C}_{s^{2} \bar{\psi} \psi}\right) \\
& =\hat{C}_{s^{2} \bar{\psi} \psi}^{(1 / N)}\left(1+\delta Z_{s^{2} \bar{\psi} \psi}+A_{\psi}+\frac{A_{s^{2}}}{2}+\delta u+\mathcal{O}\left(\frac{1}{N^{2}}\right)\right),
\end{aligned}
$$

where the leading order coefficient is given by [44]

$$
\hat{C}_{s^{2} \bar{\psi} \psi}^{(1 / N)}=\frac{1}{N} \frac{2^{d-\frac{3}{2}} \sin \left(\frac{\pi d}{2}\right) \Gamma\left(\frac{d-1}{2}\right)}{\pi^{3 / 2} \Gamma\left(\frac{d}{2}\right)},
$$

while for the $1 / N$ correction we derive

$$
\delta \hat{C}_{s^{2} \bar{\psi} \psi}=\delta Z_{s^{2} \bar{\psi} \psi}+A_{\psi}+A_{s}+\frac{h}{2}+\delta u .
$$

As a consistency check for our expression (5.5) let us consider its limiting value in $d=4$ dimension,

$$
\left.\delta \hat{C}_{s^{2} \bar{\psi} \psi}\right|_{d=4}=-\frac{6}{N},
$$

where the UV fixed point of the GN model is critically equivalent to the IR fixed point of the GNY model. For the latter, the $\delta \hat{C}_{s^{2} \bar{\psi} \mu}$ is obtained perturbatively in $\epsilon$ in $d=$ $4-\epsilon$ dimensions by using the fixed point value of the $s \bar{\psi} \psi$ coupling [11]

$g_{1}^{\star}=4 \pi \sqrt{\frac{\epsilon}{N}}\left(1-\frac{3}{N}+\mathcal{O}\left(1 / N^{2}\right)\right)+\mathcal{O}\left(1 / N^{2}, \epsilon\right)$

in the leading-order $\left\langle s^{2} \bar{\psi} \psi\right\rangle$ diagram. Since there are two $s \bar{\psi} \psi$ vertices in that diagram, we obtain $\left.\delta \hat{C}_{s^{2} \bar{\psi} \psi}\right|_{d=4}=$ $-6 / N+\mathcal{O}\left(1 / N^{2}, \epsilon\right)$, in agreement with (5.6).

Finally, notice that the normalized amplitude $\hat{C}_{s^{2} \bar{\psi} \psi}^{(1 / N)}$ vanishes in $d=2$, which can be alternatively seen as follows. In two dimensions the GN model is asymptotically free, and $s \simeq \bar{\psi} \psi$. Therefore the $\left\langle s^{2} \bar{\psi} \psi\right\rangle$ three-point function can be calculated using the diagram 


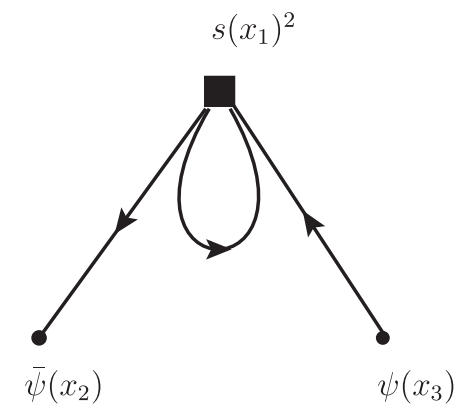

This diagram contains a fermionic tadpole loop, and therefore it vanishes in CFT. In fact, $\left.\left\langle s^{2} \bar{\psi} \psi\right\rangle\right|_{d=2}=0$ to all orders in $1 / N$.

\section{CONFORMAL TRIANGLE FROM PROPAGATOR}

In this section we will provide an alternative derivation of the $1 / N$ correction $\delta V_{s^{2} s s}$ to the amplitude of the $s^{2} s s$ conformal triangle via the $\left\langle s^{2} s^{2}\right\rangle$ propagator. This will serve as a nontrivial consistency check for our result (3.31). On the other hand, the calculation presented in this section can

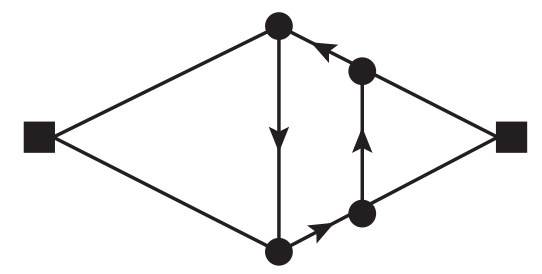

be viewed as a new method of deriving conformal triangles, which we believe has not been reported before in the literature on the large $N$ vector models.

At the leading order the $\left\langle s^{2} s^{2}\right\rangle$ two-point function is given by the diagram

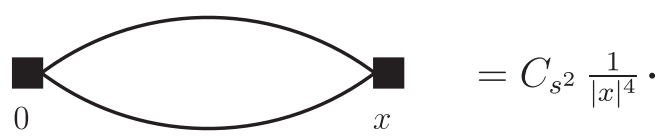

The diagrams contributing at the next-to-leading order are given by dressing of the internal $s$ lines and the $s^{2} s s$ subdiagram of this leading order diagram. The former is straightforward to calculate, ${ }^{18}$

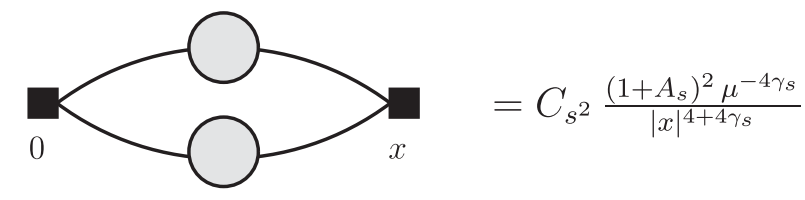

while the latter is given by

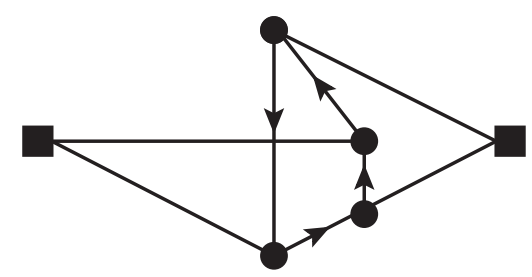

Using the $s^{2} s s$ conformal triangle introduced in Sec. III B we can rewrite the total of the diagrams contributing to $\left\langle s^{2} s^{2}\right\rangle$ up to the next-to-leading order as

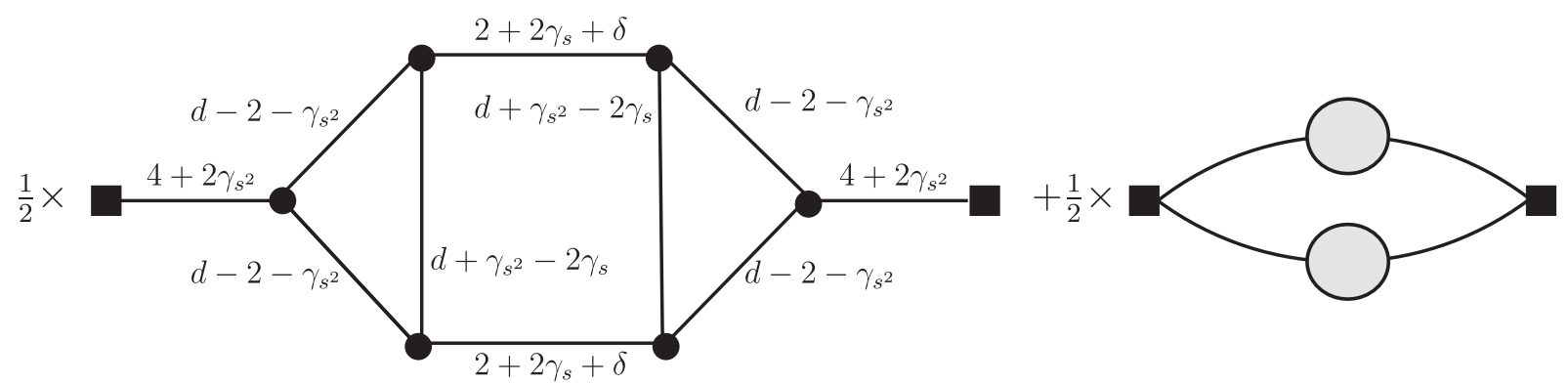

Here the first diagram contains two $s^{2} s s$ conformal triangles. To compensate for this double counting we multiplied it by the factor of $1 / 2$. Furthermore, the first diagram already contains the leading order $\left\langle s^{2} s^{2}\right\rangle$ diagram, as well as the corrections obtained by dressing of its internal $s$ lines. However since the first diagram is multiplied by the factor of $1 / 2$, we need to add another $1 / 2$ of such contributions. Finally, notice that the first diagram is divergent. To regularize it we introduced a small shift $\delta$ to the internal $s$ propagators.

\footnotetext{
${ }^{18}$ This diagram contains the entire leading order contribution to $\left\langle s^{2} s^{2}\right\rangle$.
} 
Contribution of the second diagram is given by

$$
\left\langle s^{2}(x) s^{2}(0)\right\rangle \supset C_{s^{2}} \frac{1}{|x|^{4}}\left(\frac{1}{2}+A_{s}-2 \gamma_{s} \log (\mu|x|)\right),
$$

while contribution of the first diagram is

$$
\begin{aligned}
& \left\langle s^{2}(x) s^{2}(0)\right\rangle \\
& \supset \frac{1}{2}\left(C_{s^{2}}\left(1+A_{s^{2}}\right) C_{s}\left(1+A_{s}\right) Z_{s^{2} s s}^{(0)}\left(1+\delta Z_{s^{2} s s}\right)\right)^{2} V(\delta),
\end{aligned}
$$

where $V(\delta)$ is obtained by integrating over the internal vertices. To find the latter we first integrate over the leftmost and the right-most vertices, resulting in

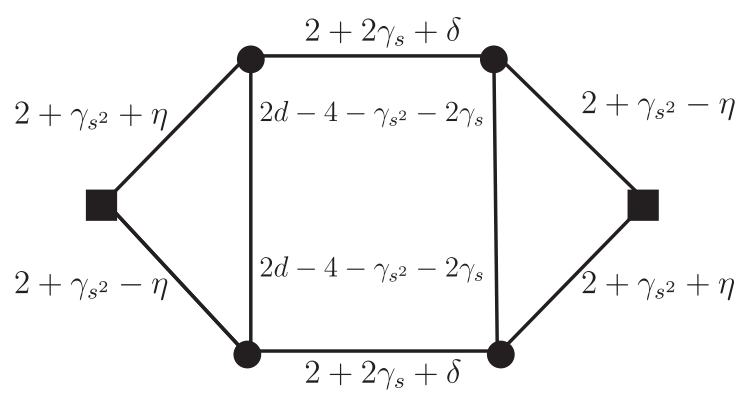

Here we have introduced an auxiliary parameter $\eta$, shifting exponents of some of the lines. One can easily see that the diagram is an even function of $\eta,{ }^{19}$ and as a result choosing $\eta=\mathcal{O}(\delta)$ will not affect the value of the diagram in the limit $\delta \rightarrow 0[14,15,49]$. We will take advantage of this fact by setting $\eta=\delta$, which will render two of the vertices unique. Integrating over those vertices we obtain the diagram

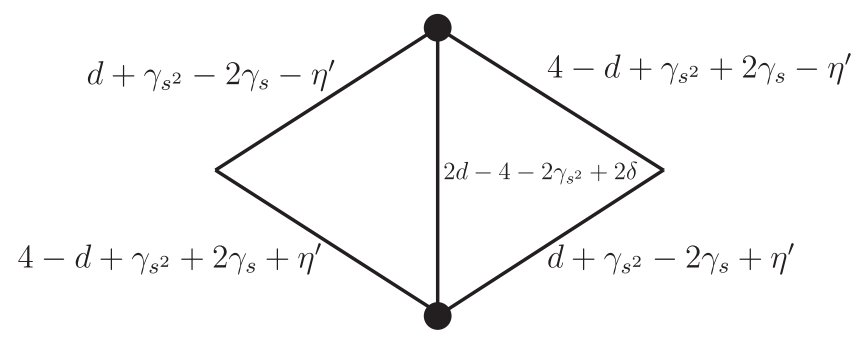

Here we introduced yet another auxiliary shift $\eta^{\prime}$, such that the resulting diagram is an even function of $\eta^{\prime} .{ }^{20}$ Consequently, choosing $\eta^{\prime}=\delta$ will not change the value of the diagram in the $\delta \rightarrow 0$ limit, while this will make the topmost vertex unique. Completing the last two integrals we obtain

$$
\begin{aligned}
V(\delta)= & \frac{1}{2} U\left(2+\gamma_{s^{2}}, \frac{d-\gamma_{s^{2}}}{2}-1, \frac{d-\gamma_{s^{2}}}{2}-1\right)^{2} U\left(1+\gamma_{s}+\frac{\delta}{2}, d-2-\gamma_{s}-\frac{\gamma_{s^{2}}}{2}, 1+\frac{\gamma_{s^{2}}-\delta}{2}\right)^{2} \\
& \times U\left(d-2-\gamma_{s^{2}}+\delta, \frac{d+\gamma_{s^{2}}-\delta}{2}-\gamma_{s}, \frac{\gamma_{s^{2}}-d-\delta}{2}+2+\gamma_{s}\right) U\left(\frac{d}{2}+\delta, \frac{d}{2}+\delta,-2 \delta\right) \frac{\mu^{-2 \gamma_{s^{2}}-2 \delta}}{|x|^{4+2 \gamma_{s^{2}}+2 \delta}},
\end{aligned}
$$

where $1 / 2$ is the symmetry factor of the diagram. Expanding the product of the $U$ functions around $\delta=0$ and $N=\infty$ we obtain

$$
\begin{aligned}
V(\delta) & =v_{0}\left(1+\frac{\gamma_{s^{2}}-2 \gamma_{s}}{\delta}+\delta v\right) \frac{\mu^{-2 \gamma_{s^{2}}-2 \delta}}{|x|^{4+2 \gamma_{s^{2}}+2 \delta}} \\
& =v_{0}\left(1+\delta v+\left(4 \gamma_{s}-4 \gamma_{s^{2}}\right) \log (\mu|x|)\right) \frac{1}{|x|^{4}}
\end{aligned}
$$

where we subtracted divergent part using $s^{2} s s$ counterterm discussed in Sec. IV, and

$$
v_{0}=\frac{C_{s^{2}}}{\left(C_{s^{2}} C_{s} Z_{s^{2} s s}^{(0)}\right)^{2}},
$$

\footnotetext{
${ }^{19}$ One can see this by noticing that $\eta \rightarrow-\eta$ can be undone by swapping vertices of integration related by mirror reflection in the horizontal axes.

${ }^{20}$ This can be seen by renaming the vertices of integration $x_{1,2}$ as $x_{1} \rightarrow x-x_{2}, x_{2} \rightarrow x-x_{1}$. We refer the reader to [49] for the detailed explanation of this method of calculating such diagrams.
}

$\delta v=\left(2 \gamma_{s}+\gamma_{s^{2}}\right)\left(\pi \cot \left(\frac{\pi d}{2}\right)+H_{d-3}\right)-2 \frac{d-6}{d-4} \gamma_{s^{2}}$.

The corresponding contribution to the two-point function is then

$$
\begin{aligned}
\left\langle s^{2}(x) s^{2}(0)\right\rangle \supset & C_{s^{2}}\left(\frac{1}{2}+A_{s^{2}}+A_{s}+\delta Z_{s^{2} s s}+\frac{\delta v}{2}\right. \\
& \left.+\left(2 \gamma_{s}-2 \gamma_{s^{2}}\right) \log (\mu|x|)\right) \frac{1}{|x|^{4}},
\end{aligned}
$$

Combining (6.1) with (6.6) we obtain

$$
\begin{aligned}
& \left\langle s^{2}(x) s^{2}(0)\right\rangle \\
& \quad=C_{s^{2}}\left(1+A_{s^{2}}+2 A_{s}+\delta Z_{s^{2} s s}+\frac{\delta v}{2}\right) \frac{1}{|x|^{4+2 \gamma_{s^{2}}}} .
\end{aligned}
$$

Using the expression (3.31) for $\delta Z_{s^{2} s s}$ derived at the nextto-leading order in $1 / N$ in Sec. III we obtain 


$$
\begin{aligned}
2 A_{s}+\delta Z_{s^{2} s s}+\frac{\delta v}{2} & =W_{3}+W_{4}-\delta w-\delta z+\frac{\delta v}{2} \\
& =\frac{\gamma_{s^{2}}}{2}-\gamma_{s}+\frac{1}{N} \frac{2^{d} \sin \left(\frac{\pi d}{2}\right) \Gamma\left(\frac{d+1}{2}\right)}{\pi^{3 / 2} \Gamma\left(\frac{d}{2}\right)} .
\end{aligned}
$$

Using expressions (2.5) and (4.18) for the anomalous dimensions $\gamma_{s, s^{2}}$ we can verify explicitly that the rhs of (6.8) vanishes, which provides a nontrivial consistency check for our calculation of the $W_{3}+W_{4}$ diagrams performed in Sec. III, as well as for the resulting value of $\delta Z_{s^{2} s s}$.

\section{DISCUSSION}

In this paper we derived new CFT data at the UV fixed point of the GN model in $2<d<4$ dimensions. In particular, we further established the computational power of the background field method, first proposed in the context of the large- $N$ vector models in [44], for the calculation of the finite parts of the effective vertices, and the corresponding OPE coefficients. To this end, we derived new expressions for the $s^{2} \bar{\psi} \psi$ and $s^{2} s s$ conformal triangles, and obtained the correlation functions $\left\langle s^{2} \bar{\psi} \psi\right\rangle$, $\left\langle s^{2} s s\right\rangle$, and $\left\langle s^{2} s^{2}\right\rangle$, while working at the next-to-leading order in the $1 / N$ expansion.

Our results are complementary to the literature on the vector models, in that they provide the finite parts of the correlation functions, useful to obtain the OPE coefficients. Specifically, the background field method allows one to easily go beyond the singular parts of the correlation functions, and the associated anomalous dimensions of the primary operators of the theory.

A natural extension of our results would be to apply the methods developed in the present work to the $O(N)$ vector model and derive the corresponding conformal triangles $s^{2} s s$ and $s^{2} \phi \phi$, where $\phi$ is the fundamental $O(N)$ field and $s$ is the Hubbard-Stratonovich field. In particular, the $s^{2} s s$ conformal triangle can be subjected to the consistency check by calculating the $\left\langle s^{2} s^{2}\right\rangle$ two-point function, along the lines of Sec. VI. One can also use this data to derive the $\left\langle s^{2} s s\right\rangle$ and $\left\langle s^{2} \phi \phi\right\rangle$ three-point functions and extract the corresponding OPE coefficients.

Another possible application of our results could be found in the study of vector models at finite temperature. This direction of research has recently received a renewed attention due to the discovery of the biconical vector models exhibiting symmetry breaking at all temperatures [50-52].

\section{ACKNOWLEDGMENTS}

We thank Michael Smolkin for numerous discussions, comments on the draft, and the suggestion to calculate $\left\langle s^{2} \bar{\psi} \psi\right\rangle$, and John Gracey for helpful correspondence. This work was partially supported by the Binational Science Foundation (Grant No. 2016186), the Israeli Science Foundation Center of Excellence (Grant No. 2289/18) and by the Quantum Universe I-CORE program of the Israel Planning and Budgeting Committee (Grant No. 1937/12).
[1] A. M. Polyakov, Conformal symmetry of critical fluctuations, JETP Lett. 12, 381 (1970), http://jetpletters.ru/ps/ 1737/article_26381.shtml.

[2] G. Parisi, On self-consistency conditions in conformal covariant field theory, Lett. Nuovo Cimento 4, 777 (1972).

[3] A. M. Polyakov, Nonhamiltonian approach to conformal quantum field theory, Zh. Eksp. Teor. Fiz. 66, 23 (1974), http://www.jetp.ac.ru/cgi-bin/dn/e_039_01_0010.pdf.

[4] S. Ferrara, A. F. Grillo, and R. Gatto, Tensor representations of conformal algebra and conformally covariant operator product expansion, Ann. Phys. (N.Y.) 76, 161 (1973).

[5] S. El-Showk, M. F. Paulos, D. Poland, S. Rychkov, D. Simmons-Duffin, and A. Vichi, Solving the 3D Ising model with the conformal bootstrap, Phys. Rev. D 86, 025022 (2012).

[6] D. Simmons-Duffin, The conformal bootstrap, arXiv: 1602.07982.

[7] H. E. Stanley, Dependence of Critical Properties on Dimensionality of Spins, Phys. Rev. Lett. 20, 589 (1968).

[8] R. Guida and J. Zinn-Justin, Critical exponents of the N vector model, J. Phys. A 31, 8103 (1998).
[9] K. G. Wilson and M. E. Fisher, Critical Exponents in 3.99 Dimensions, Phys. Rev. Lett. 28, 240 (1972).

[10] D. J. Gross and A. Neveu, Dynamical symmetry breaking in asymptotically free field theories, Phys. Rev. D 10, 3235 (1974).

[11] J. Zinn-Justin, Four fermion interaction near fourdimensions, Nucl. Phys. B367, 105 (1991).

[12] J. Braun, Fermion interactions and universal behavior in strongly interacting theories, J. Phys. G 39, 033001 (2012).

[13] G. Parisi, The theory of nonrenormalizable interactions. 1. The large N expansion, Nucl. Phys. B100, 368 (1975).

[14] A. N. Vasiliev, Y. M. Pismak, and Y. R. Khonkonen, Simple method of calculating the critical indices in the $1 / \mathrm{N}$ expansion, Theor. Math. Phys. 46, 104 (1981).

[15] A. N. Vasiliev, Y. M. Pismak, and Y. R. Khonkonen, 1/N expansion: Calculation of the exponents $\eta$ and $\mathrm{Nu}$ in the Order $1 / \mathrm{N}^{2}$ for arbitrary number of dimensions, Theor. Math. Phys. 47, 465 (1981).

[16] A. N. Vasiliev and M. Y. Nalimov, Analog of dimensional regularization for calculation of the renormalization group functions in the $1 / \mathrm{n}$ expansion for arbitrary dimension of space, Theor. Math. Phys. 55, 423 (1983). 
[17] A. N. Vasiliev, Y. M. Pismak, and Y. R. Khonkonen, 1/N expansion: Calculation of the exponent eta in the order $1 / \mathrm{N}^{3}$ by the conformal bootstrap method, Theor. Math. Phys. 50, 127 (1982).

[18] J. A. Gracey, Calculation of exponent eta to $\mathrm{O}\left(1 / \mathrm{N}^{2}\right)$ in the O(N) Gross-Neveu model, Int. J. Mod. Phys. A 06, 395 (1991); , Erratum, Int. J. Mod. Phys. A 06, 2755 (1991).

[19] K. Lang and W. Ruhl, The Critical O(N) sigma model at dimension $2<d<4$ and order $1 / \mathrm{n}^{2}$ : Operator product expansions and renormalization, Nucl. Phys. B377, 371 (1992).

[20] J. A. Gracey, Anomalous mass dimension at $\mathrm{O}\left(1 / \mathrm{N}^{2}\right)$ in the O(N) Gross-Neveu model, Phys. Lett. B 297, 293 (1992).

[21] A. N. Vasiliev, S. E. Derkachov, N. A. Kivel, and A. S. Stepanenko, The 1/n expansion in the Gross-Neveu model: Conformal bootstrap calculation of the index eta in order $1 / \mathrm{n}^{3}$, Theor. Math. Phys. 94, 127 (1993).

[22] A. N. Vasiliev and A. S. Stepanenko, The $1 / \mathrm{n}$ expansion in the Gross-Neveu model: Conformal bootstrap calculation of the exponent $1 / \mathrm{nu}$ to the order $1 / \mathrm{n}^{2}$, Theor. Math. Phys. 97, 1349 (1993).

[23] J. A. Gracey, Computation of Beta-prime $(\mathrm{g}(\mathrm{c}))$ at $\mathrm{O}\left(1 / \mathrm{N}^{2}\right)$ in the $\mathrm{O}(\mathrm{N})$ Gross-Neveu model in arbitrary dimensions, Int. J. Mod. Phys. A 09, 567 (1994).

[24] J.A. Gracey, Computation of critical exponent eta at $\mathrm{O}\left(1 / \mathrm{N}^{3}\right)$ in the four Fermi model in arbitrary dimensions, Int. J. Mod. Phys. A 09, 727 (1994).

[25] K. Lang and W. Ruhl, Critical nonlinear O(N) sigma models at $2<d<4$ : The Degeneracy of quasiprimary fields and it resolution, Z. Phys. C 61, 495 (1994).

[26] A. C. Petkou, C(T) and C(J) up to next-to-leading order in $1 / \mathrm{N}$ in the conformally invariant $\mathrm{O}(\mathrm{N})$ vector model for $2<d<4$, Phys. Lett. B 359, 101 (1995).

[27] A. Petkou, Conserved currents, consistency relations and operator product expansions in the conformally invariant O(N) vector model, Ann. Phys. (N.Y.) 249, 180 (1996).

[28] S. E. Derkachov, N. A. Kivel, A. S. Stepanenko, and A. N. Vasiliev, On calculation in $1 / n$ expansions of critical exponents in the Gross-Neveu model with the conformal technique, arXiv:hep-th/9302034.

[29] S. E. Derkachov and A. N. Manashov, The simple scheme for the calculation of the anomalous dimensions of composite operators in the $1 / \mathrm{N}$ expansion, Nucl. Phys. B522, 301 (1998).

[30] T. Leonhardt and W. Ruhl, The minimal conformal $\mathrm{O}(\mathrm{N})$ vector sigma model at $d=3$, J. Phys. A 37, 1403 (2004).

[31] L. Fei, S. Giombi, and I. R. Klebanov, Critical $O(N)$ models in 6- $\epsilon$ dimensions, Phys. Rev. D 90, 025018 (2014).

[32] L. Fei, S. Giombi, I. R. Klebanov, and G. Tarnopolsky, Three loop analysis of the critical $\mathrm{O}(\mathrm{N})$ models in $6-\epsilon$ dimensions, Phys. Rev. D 91, 045011 (2015).

[33] J. A. Gracey, Four loop renormalization of $\phi^{3}$ theory in six dimensions, Phys. Rev. D 92, 025012 (2015).
[34] A. N. Manashov and E. D. Skvortsov, Higher-spin currents in the Gross-Neveu model at $1 / \mathrm{n}^{2}, \mathrm{~J}$. High Energy Phys. 01 (2017) 132.

[35] K. Diab, L. Fei, S. Giombi, I. R. Klebanov, and G. Tarnopolsky, On $C_{J}$ and $C_{T}$ in the Gross-Neveu and $\mathrm{O}$ (N) models, J. Phys. A 49, 405402 (2016).

[36] S. Giombi, G. Tarnopolsky, and I. R. Klebanov, On $C_{J}$ and $C_{T}$ in conformal QED, J. High Energy Phys. 08 (2016) 156.

[37] L. Fei, S. Giombi, I. R. Klebanov, and G. Tarnopolsky, Yukawa CFTs and emergent supersymmetry, Prog. Theor. Exp. Phys. 2016, 12C105 (2016).

[38] J. A. Gracey, T. Luthe, and Y. Schroder, Four loop renormalization of the Gross-Neveu model, Phys. Rev. D 94, 125028 (2016).

[39] A. N. Manashov and M. Strohmaier, Correction exponents in the Gross-Neveu-Yukawa model at $1 / \mathrm{N}^{2}$, Eur. Phys. J. C 78, 454 (2018).

[40] J. A. Gracey, Large $N_{f}$ quantum field theory, Int. J. Mod. Phys. A 33, 1830032 (2018).

[41] L. F. Alday, J. Henriksson, and M. van Loon, An alternative to diagrams for the critical $\mathrm{O}(\mathrm{N})$ model: Dimensions and structure constants to order $1 / \mathrm{N}^{2}$, J. High Energy Phys. 01 (2020) 063.

[42] S. Giombi, R. Huang, I. R. Klebanov, S. S. Pufu, and G. Tarnopolsky, The $O(N)$ Model in $4<d<6$ : Instantons and complex CFTs, Phys. Rev. D 101, 045013 (2020).

[43] M. Goykhman and M. Smolkin, Vector model in various dimensions, Phys. Rev. D 102, 025003 (2020).

[44] M. Goykhman, V. Rosenhaus, and M. Smolkin, The background field method and critical vector models, J. High Energy Phys. 02 (2021) 074.

[45] B. Rosenstein, B. J. Warr, and S. H. Park, The Four Fermi Theory Is Renormalizable in $(2+1)$-Dimensions, Phys. Rev. Lett. 62, 1433 (1989).

[46] J. Goldstone, A. Salam, and S. Weinberg, Broken symmetries, Phys. Rev. 127, 965 (1962).

[47] M. D'Eramo, G. Parisi, and L. Peliti, Theoretical predictions for critical exponents at the lambda point of Bose liquids, Lett. Nuovo Cimento 2, 878 (1971).

[48] K. Symanzik, On calculations in conformal invariant field theories, Lett. Nuovo Cimento 3, 734 (1972).

[49] S. S. Gubser, C. Jepsen, S. Parikh, and B. Trundy, O(N) and $\mathrm{O}(\mathrm{N})$ and $\mathrm{O}(\mathrm{N})$, J. High Energy Phys. 11 (2017) 107.

[50] N. Chai, S. Chaudhuri, C. Choi, Z. Komargodski, E. Rabinovici, and M. Smolkin, Symmetry Breaking at All Temperatures, Phys. Rev. Lett. 125, 131603 (2020).

[51] N. Chai, S. Chaudhuri, C. Choi, Z. Komargodski, E. Rabinovici, and M. Smolkin, Thermal order in conformal theories, Phys. Rev. D 102, 065014 (2020).

[52] N. Chai, E. Rabinovici, R. Sinha, and M. Smolkin, The bi-conical vector model at 1/N, arXiv:2011.06003. 